\title{
Positive cross-correlations due to dynamical channel blockade in a three-terminal quantum dot
}

\author{
A. Cottet, W. Belzig, and C. Bruder \\ Department of Physics and Astronomy, University of Basel, Klingelbergstrasse 82, 4056 Basel, Switzerland
}

(Received 19 March 2004; revised manuscript received 1 June 2004; published 22 September 2004)

\begin{abstract}
We investigate current fluctuations in a three-terminal quantum dot in the sequential tunneling regime. In the voltage-bias configuration chosen here, the circuit is operated as a beam splitter, i.e., one lead is used as an input and the other two as outputs. In the limit where a double occupancy of the dot is not possible, a super-Poissonian Fano factor of the current in the input lead and positive cross correlations between the current fluctuations in the two output leads can be obtained, due to dynamical channel blockade. When a single orbital of the dot transports current, these effects can be obtained by lifting the spin degeneracy of the circuit with ferromagnetic leads or with a magnetic field. When several orbitals participate in the electronic conduction, lifting spin degeneracy is not necessary. In all cases, we show that a super-Poissonian Fano factor for the input current is not equivalent to positive cross correlations between the outputs. We identify the conditions for obtaining these two effects and discuss possible experimental realizations.
\end{abstract}

PACS number(s): 73.23. $-\mathrm{b}, 72.70 .+\mathrm{m}, 72.25 . \mathrm{Rb}$

\section{INTRODUCTION}

The study of current noise in mesoscopic circuits has become a central subfield of mesoscopic physics because it allows us to access information not available through measurements of the average currents (for reviews, see Refs. 1 and 2). Current fluctuations can first be probed through the autocorrelations of the current fluctuations in one branch of the circuit. For noninteracting conductors with open channels, the fermionic statistics of electrons result in a suppression of these autocorrelations below the Poisson limit. ${ }^{3-5}$ In a multiterminal circuit, current fluctuations can also be probed through the cross correlations between two different branches. Büttiker has shown that in a noninteracting electronic circuit, the zero-frequency current cross correlations are always negative provided the leads of the circuit are thermal reservoirs maintained at constant voltage potentials. ${ }^{6}$ On the experimental side, negative cross correlations have been measured very recently by Henny et al. ${ }^{7}$ and Oliver et al. ${ }^{8}$ in mesoscopic beam splitters. Oberholzer et al. have shown how the cross correlations vanish in the classical limit. ${ }^{9}$

Up to now, positive cross correlations have never been measured in electronic circuits. However, nothing forbids to reverse the sign of cross correlations if a hypothesis of Büttiker's proof is not fulfilled (see Ref. 10 for a recent review). First, it has been shown theoretically that positive cross correlations can be obtained in an electronic circuit by relaxing the hypotheses of Büttiker regarding the leads, for instance, by taking one of the leads superconducting ${ }^{11-22}$ or by using leads with an imperfect ${ }^{23}$ or time-dependent ${ }^{24}$ voltage bias. Positive cross correlations are also expected at finite frequencies, due to the plasmonic screening currents existing in capacitive circuits. ${ }^{10,25}$ It follows from Büttiker's work that obtaining positive cross correlations at zero frequency without modifying the assumptions on the leads requires to have interactions inside the device. Safi et al. have considered a two-dimensional electron gas in the fractional quantum Hall regime, described by a chiral Luttinger liquid theory. ${ }^{26}$ Zerofrequency positive cross correlations can be obtained in this system in the limit of small filling factors, where the excita- tions of the chiral Luttinger liquid take a bosonic character. This leaves open the question whether interactions localized inside the beam splitter can lead to zero-frequency positive cross correlations even for a normal fermionic circuit.

Current correlations in a single quantum dot have been studied in the sequential tunneling limit, ${ }^{27-30}$ in the cotunneling regime, ${ }^{31,32}$ and in the Kondo regime. ${ }^{33}$ In the (spindegenerate) sequential tunneling limit, a sub-Poissonian Fano factor has been found for some two-terminal cases ${ }^{27-29}$ and, for the three-terminal case, cross correlations are expected to be always negative when the intrinsic level spacing $\Delta E$ of the dot is much smaller than temperature. ${ }^{28}$ However, a super-Poissonian Fano factor has been predicted for a twoterminal quantum dot with $\Delta E \gg k_{B} T$ connected to ferromagnetic leads. ${ }^{30}$ In the cotunneling regime, a super-Poissonian Fano factor can be obtained in the two-terminal case. ${ }^{31}$ The extent to which this would lead to positive cross-correlations for a three-terminal quantum dot was not clear.

This led us to consider, in Refs. 34 and 35, the case of a three-terminal quantum dot with $\Delta E \gg k_{B} T$, operated as a beam splitter: one contact acts as source and the other two as drains. We have assumed that only one orbital of the dot, i.e., one single-particle level, transports current, and that Coulomb interactions prevent a double occupancy of this orbital. We have considered both the Fano factor $F_{2}$ in the input lead, called the input Fano factor, and the cross correlations $S_{13}(\omega)$ between the two output leads, called output cross correlations. We have proposed two different methods to obtain a super-Poissonian $F_{2}$ or $S_{13}(\omega=0)>0$ in this system, in the sequential tunneling limit. Both methods rely on lifting spin degeneracy, either by using ferromagnetic leads ${ }^{34}$ or by using paramagnetic leads and applying a magnetic field to the dot. ${ }^{35}$ Note that in these works, the leads are biased with constant voltages and modeled as noninteracting Fermi gases. Then, with respect to Büttiker's proof, only the hypothesis of the absence of interactions inside the device itself is relaxed. Moreover, in contrast to the system studied in Ref. 26, excitations inside the device remain purely fermionic. Our works ${ }^{34,35}$ give a positive answer to the question whether 
TABLE I. Summary of the different cases of quantum dot circuits treated in this article. All these circuits have the geometry shown in Fig. 1. They differ in the number of dot orbitals implied in the current transport, the magnetic field $B$ applied to the dot, and the magnetic polarizations $P_{i}$ of the leads. In the cases marked by a cross, a super-Poissonian $F_{2}$ or a positive $S_{13}(\omega=0)$ can be obtained for appropriate values of the bias voltage $V$, the polarizations $P_{i}$, and the tunnel rates $\gamma_{i}$. In the one-orbital paramagnetic case, the position $E_{0}$ of the dot orbital level with respect to the zero-bias Fermi level is also critical. Note that a super-Poissonian $F_{2}$ is not automatically associated with $S_{13}(\omega=0)>0$.

\begin{tabular}{ccccccc}
\hline \hline Case treated in section & II & III & IV & IV & IV & V \\
\hline Number of dot orbitals & 1 & 1 & 1 & 1 & 1 & 2 \\
Lead polarizations $P_{i}$ & 0 & $\neq 0$ & 0 & 0 & $\neq 0$ & 0 \\
$\quad$ Magnetic field $B$ & 0 & 0 & $\neq 0$ & $\neq 0$ & $\neq 0$ & 0 \\
Orbital level position $E_{0}$ & & & $>0$ & $<0$ & & \\
Possibility of $S_{13}(\omega=0)>0$ & & & & & & \\
$\quad$ or super-Poissonian $F_{2}$ & & & & & & \\
Below the high-voltage limit: & & $\times$ & & $\times$ & $\times$ & $\times$ \\
In the high-voltage limit: & & $\times$ & & & $\times$ & $\times$ \\
\hline \hline
\end{tabular}

zero-frequency positive cross correlations can occur in a perfectly voltage-biased normal fermionic circuit. They nevertheless leave open the question whether lifting spin degeneracy is necessary to do so. Eventually, it appears in Refs. 34 and 35 that for certain cases, a super-Poissonian $F_{2}$ can be obtained without a positive $S_{13}(\omega=0)$. This calls for a thorough analysis of the relation between $F_{2}$ and $S_{13}(\omega=0)$.

To this end, in this article, we investigate in detail the physical origin of the positive cross correlations found in Refs. 34 and 35. The essential ingredient is the existence of Coulomb interactions on the dot. (Note that in a spin valve connected to ferromagnetic leads, in which there are no charging effects, the cross correlations where found to be negative. $)^{36}$ In the limit where only one (singly occupied) orbital level of the dot transports current, positive cross correlations are caused by a mechanism of dynamical spin blockade which can occur when spin degeneracy is lifted. Simply speaking, up and down spins tunnel through the dot with different rates. Due to the Coulomb interaction, the spins which tunnel with a lower rate modulate the transport through the opposite spin channel, leading to a bunching of tunneling events. We show how this bunching can lead to a super-Poissonian $F_{2}$ or a positive $S_{13}(\omega=0)$ ). (Table I gives a summary of the conditions for which these properties can be obtained.) In the limit of equal polarization of the output leads and high bias voltage, the electronic transport is unidirectional and the division of current between the two outputs is the same for the two spin directions. This leads in the one-orbital case to a simple relation [see Eq. (25)] between $F_{2}$ and $S_{13}(\omega=0)$ : a super-Poissonian $F_{2}$ is automatically associated with a positive $S_{13}(\omega=0)$. However, in general, this relation is not fulfilled even for a one-orbital dot. In particular, in the case where the leads of the one-orbital dot are paramagnetic and where spin degeneracy is lifted by a magnetic field, a positive $S_{13}(\omega=0)$ can only be obtained in an intermediary voltage range where relation (25) is not valid.

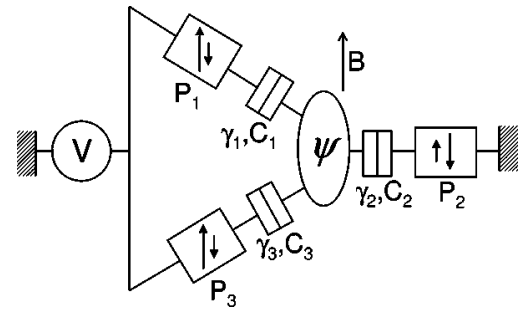

FIG. 1. Electrical diagram of a quantum dot connected to three leads $i \in\{1,2,3\}$ with collinear magnetic polarizations $P_{i}$, through tunnel junctions with net tunneling rates $\gamma_{i}$ and capacitances $C_{i}$. A bias voltage $V$ is applied to leads 1 and 3 ; lead 2 is connected to ground. A magnetic field $B$ collinear to the lead polarizations is applied to the dot.

This paper supplements the study of the one orbital case with an analysis of the effect of the position of the dot orbital level with respect to the zero-bias Fermi level. In the paramagnetic case, this parameters turns out to be critical to get a positive $S_{13}(\omega=0)$. An analysis of the frequency dependence of $S_{13}(\omega)$ is provided. We also show that $S_{13}(\omega=0)>0$ can persist in the one orbital case when the leads are magnetically polarized and a magnetic field applied. Eventually, we show that there is a direct mapping between this last case and that of a spin-degenerate quantum dot with two orbital levels transporting current. This mapping suggests a new way to get positive cross correlations. In this spin-degenerate twoorbital case, positive cross correlations stem from the partial blockade of an electronic channel by another one, thus we propose to call this effect "dynamical channel blockade." This result demonstrates that lifting spin degeneracy is not necessary for obtaining zero-frequency positive cross correlations due to interactions inside a beam splitter device, even for a normal fermionic circuit with a perfect voltage bias.

The present article is organized as follows. Section II develops the mathematical description valid for the one-orbital problem. This one-orbital problem is analyzed for two different configurations. First, the case of ferromagnetic leads and zero magnetic field is treated in Sec. III. Secondly, the case of a Zeeman splitting created by a magnetic field is treated in Sec. IV. In Sec. V, we show how to map the twoorbital spin-degenerate problem onto the one-orbital problem.

\section{MODEL AND GENERAL DESCRIPTION FOR THE ONE-ORBITAL CASE}

\section{A. Model}

We consider a quantum dot connected to three leads $i$ $\in\{1,2,3\}$, through tunnel junctions with capacitances $C_{i}$ and net spin-independent tunneling rates $\gamma_{i}$ (Fig. 1). The leads are magnetically polarized in collinear directions. We also assume that the dot is subject to a magnetic field $B$ collinear to the lead polarizations. A voltage bias $V$ is applied to leads 1 and 3 whereas lead 2 is connected to ground. The voltage $V$ is considered as positive, such that it is energetically more favorable for electrons to go from the input electrode 2 to the output electrodes 1 or 3 than in the opposite direction. In this section, we also assume that 


$$
k_{B} T, \mu_{B} B, e V \ll E_{C}, \Delta E,
$$

where the charging energy $E_{C}=e^{2} / 2 C$ of the dot depends on $C=\Sigma_{i} C_{i}$, and where $\Delta E$ is the intrinsic level spacing of the dot. According to Eq. (1), only one orbital level of the dot, with energy $E_{0}$, needs to be taken into account to describe the current transport, and this level cannot be doubly occupied. In this situation, there are three possible states $\psi$ for the dot: either empty, i.e., $\psi=0$, or occupied with one electron with spin $\sigma \in\{\uparrow, \downarrow\}$, i.e., $\psi=\sigma$. The magnetic field $B$ induces a Zeeman splitting of the level according to $E_{\downarrow(\uparrow)}=E_{0}+($ -) $g \mu_{B} B / 2$, where $\mu_{B}=e \hbar / 2 m$ is the Bohr magneton. In this article, we will assume $B \geqslant 0$, i.e., the up-spin level is energetically lower than the down-spin level in the presence of a magnetic field. The collinear magnetic polarizations $P_{j}$ of the leads are taken into account by using spin-dependent tunneling rates $\gamma_{j \uparrow}=\gamma_{j}\left(1+P_{j}\right)$ and $\gamma_{j \downarrow}=\gamma_{j}\left(1-P_{j}\right)$. In a simple model, the spin dependence is a consequence of the different densities of states for electrons with up and down spins in the leads. ${ }^{37}$ The rate for an electron to tunnel on/off the dot $(\epsilon$ $=+/-1)$ through junction $j$ is then given by

$$
\Gamma_{j \sigma}^{\epsilon}=\gamma_{j \sigma} /\left\{1+\exp \left[\epsilon\left(E_{\sigma}-e V_{j}\right) / k_{B} T\right]\right\},
$$

where $V_{1}=V_{3}=-C_{2} V / C$ and $V_{2}=\left(C_{1}+C_{3}\right) V / C$. Here, we took the Fermi energy $E_{F}=0$ for lead 2 as a reference. On the dot, there can be spin-flip scattering, due for instance to spinorbit coupling or to magnetic impurities. According to the detailed balance rule, we write the spin-flip rates as

$$
\Gamma_{\uparrow \downarrow}=\gamma_{s f} \exp \left(+\frac{g \mu_{B} B}{2 k_{B} T}\right)
$$

for the $\downarrow \rightarrow \uparrow$ transition and

$$
\Gamma_{\downarrow \uparrow}=\gamma_{s f} \exp \left(-\frac{g \mu_{B} B}{2 k_{B} T}\right)
$$

for the $\uparrow \rightarrow \downarrow$ transition.

\section{B. Master equation treatment}

In the sequential-tunneling limit $\hbar \gamma_{j \sigma} \ll k_{B} T$, electronic transport through the dot can be described by the master equation $^{27}$

$$
\frac{d}{d t}\left[\begin{array}{l}
p_{\uparrow}(t) \\
p_{\downarrow}(t) \\
p_{0}(t)
\end{array}\right]=\hat{M}\left[\begin{array}{l}
p_{\uparrow}(t) \\
p_{\downarrow}(t) \\
p_{0}(t)
\end{array}\right],
$$

where $p_{\psi}(t), \psi \in\{\uparrow, \downarrow, 0\}$, is the instantaneous occupation probability of state $\psi$ at time $t$, and where

$$
\hat{M}=\left[\begin{array}{ccc}
-\Gamma_{\uparrow}^{-}-\Gamma_{\downarrow \uparrow} & \Gamma_{\uparrow \downarrow} & \Gamma_{\uparrow}^{+} \\
\Gamma_{\downarrow \uparrow} & -\Gamma_{\downarrow}^{-}-\Gamma_{\uparrow \downarrow} & \Gamma_{\downarrow}^{+} \\
\Gamma_{\uparrow}^{-} & \Gamma_{\downarrow}^{-} & -\Gamma_{\uparrow}^{+}-\Gamma_{\downarrow}^{+}
\end{array}\right]
$$

depends on the total rates $\Gamma_{\sigma}^{\epsilon}=\Sigma_{j} \Gamma_{j \sigma}^{\epsilon}$. This master equation treatment relies on a Markovian approximation valid for frequencies $\omega$ lower than $\max \left[k_{B} T, \min _{\sigma, i}\left(\left|E_{\sigma}-e V_{i}\right|\right)\right] / \hbar .{ }^{38}$ From Eq. (3), the stationary occupation probabilities $\bar{p}_{\psi}$ are

$$
\bar{p}_{\sigma}=\frac{\Gamma_{\sigma}^{+} \Gamma_{-\sigma}^{-}+\Gamma_{\sigma,-\sigma}\left(\Gamma_{\uparrow}^{+}+\Gamma_{\downarrow}^{+}\right)}{\gamma_{\uparrow} \gamma_{\downarrow}-\Gamma_{\uparrow}^{+} \Gamma_{\downarrow}^{+}+\sum_{\sigma^{\prime}}\left(\gamma_{\sigma^{\prime}}+\Gamma_{-\sigma^{\prime}}^{+}\right) \Gamma_{\sigma^{\prime},-\sigma^{\prime}}},
$$

with $\gamma_{\sigma}=\Sigma_{j} \gamma_{j \sigma}$, for $\sigma \in\{\uparrow, \downarrow\}$, and

$$
\bar{p}_{0}=1-\bar{p}_{\uparrow}-\bar{p}_{\downarrow} .
$$

These probabilities can be used to calculate the average value $\left\langle I_{j}\right\rangle$ of the tunneling current $I_{j}(t)$ through junction $j$ as $\left\langle I_{j}\right\rangle=\Sigma_{\sigma}\left\langle I_{j, \sigma}\right\rangle$, where $\left\langle I_{j, \sigma}\right\rangle=\Sigma_{\epsilon}\left\langle I_{j, \sigma}^{\epsilon}\right\rangle$ is the average current of electrons with spins $\sigma$, and

$$
\left\langle I_{j, \sigma}^{\epsilon}\right\rangle=e \epsilon \Gamma_{j \sigma}^{\epsilon} \bar{p}_{A(\sigma,-\epsilon)} .
$$

Here, $A(\sigma, \varepsilon)$ is the state of the dot after the tunneling of an electron with spin $\sigma$ in the direction $\epsilon$, i.e., $A(\sigma,-1)=0$ and $A(\sigma,+1)=\sigma$.

The frequency spectrum of the noise correlations can be defined as

$$
S_{i j}(\omega)=\int_{-\infty}^{+\infty} d t \mathcal{C}_{i j}(t) \exp (i \omega t)
$$

where

$$
\mathcal{C}_{i j}(t)=\left\langle\Delta I_{i}(t) \Delta I_{j}(0)\right\rangle+\left\langle\Delta I_{i}(0) \Delta I_{j}(t)\right\rangle
$$

and $\Delta I_{i}(t)=I_{i}(t)-\left\langle I_{i}\right\rangle$. Following the method developed in Ref. 27, we can write this spectrum as

$$
S_{i j}(\omega)=\delta_{i j} S_{j}^{\mathrm{Sch}}+S_{i j}^{c}(\omega),
$$

with $S_{j}^{\text {Sch }}=\Sigma_{\sigma} S_{j \sigma}^{\text {Sch }}$ and $S_{i j}^{c}(\omega)=\Sigma_{\sigma, \sigma^{\prime}} S_{i \sigma, j \sigma^{\prime}}^{c}(\omega)$. Here,

$$
S_{j \sigma}^{\mathrm{Sch}}=2 e \sum_{\epsilon}\left|\left\langle I_{j, \sigma}^{\epsilon}\right\rangle\right|
$$

is the Schottky noise associated to the tunneling of electrons with spin $\sigma$ through junction $j$ and, from Eq. (3),

$$
\begin{aligned}
\frac{S_{i \sigma, j \sigma^{\prime}}^{c}(\omega)}{2 e^{2}}= & \sum_{\epsilon, \epsilon^{\prime}} \epsilon \epsilon^{\prime}\left[\Gamma_{i \sigma}^{\epsilon^{\prime}} \hat{\mathcal{G}}_{A\left(\sigma,-\epsilon^{\prime}\right), A\left(\sigma^{\prime}, \epsilon\right)}(\omega) \Gamma_{j \sigma^{\prime}}^{\epsilon} \bar{p}_{A\left(\sigma^{\prime},-\epsilon\right)}\right. \\
& \left.+\Gamma_{j \sigma^{\prime}}^{\epsilon^{\prime}} \hat{\mathcal{G}}_{A\left(\sigma^{\prime},-\epsilon^{\prime}\right), A(\sigma, \epsilon)}(-\omega) \Gamma_{i \sigma}^{\epsilon} \bar{p}_{A(\sigma,-\epsilon)}\right],
\end{aligned}
$$

with

$$
\hat{\mathcal{G}}(\omega)=-\operatorname{Re}\left[(i \omega \hat{I}+\hat{M})^{-1}\right]
$$

and $\hat{I}$ the identity matrix. We also define, for later use, the spin components of $S_{i j}(\omega)$ :

$$
S_{i \sigma, j \sigma^{\prime}}(\omega)=\delta_{i j} \delta_{\sigma \sigma^{\prime}} S_{i \sigma}^{S c h}+S_{i \sigma, j \sigma^{\prime}}^{c}(\omega) .
$$

Due to the existence of the stationary solution $\hat{M} \bar{p}_{0}=0$, the matrix $\hat{M}$ has only two nonzero eigenvalues $\lambda_{+}$and $\lambda_{-}$, i.e., $\hat{M} v_{ \pm}=\lambda_{ \pm} v_{ \pm}$, given by

$$
\lambda_{ \pm}=\frac{1}{2}\left(-\Lambda \pm \sqrt{\Lambda^{2}-4 \Theta}\right)<0,
$$

with 


$$
\Lambda=\Gamma_{\uparrow \downarrow}+\Gamma_{\downarrow \uparrow}+\gamma_{\uparrow}+\gamma_{\downarrow}
$$

and

$$
\Theta=\gamma_{\uparrow} \gamma_{\downarrow}+\Gamma_{\uparrow \downarrow}\left(\Gamma_{\downarrow}^{+}+\gamma_{\uparrow}\right)+\Gamma_{\downarrow \uparrow}\left(\gamma_{\downarrow}+\Gamma_{\uparrow}^{+}\right)-\Gamma_{\uparrow}^{+} \Gamma_{\downarrow}^{+} .
$$

Then, the matrix $\hat{M}$ can be written in the form $\hat{M}$ $=\hat{R}^{-1}\left(\lambda_{+} \hat{E}_{+}+\lambda_{-} \hat{E}_{-}\right) \hat{R}$, where $\hat{R}$ is a reversible $3 \times 3$ matrix, and $\hat{E}_{+(-)}$is a $3 \times 3$ matrix with the element 1 at the first (second) row and first (second) column. Accordingly, $\hat{\mathcal{G}}(\omega)$ can be written as

$$
\hat{\mathcal{G}}(\omega)=\frac{\hat{\mathcal{A}}^{+}}{\omega^{2}+\lambda_{+}^{2}}+\frac{\hat{\mathcal{A}}^{-}}{\omega^{2}+\lambda_{-}^{2}},
$$

with $\hat{\mathcal{A}}^{ \pm}=-\lambda_{ \pm} \hat{R}^{-1} \hat{E}_{ \pm} \hat{R}$. Therefore, we have

$$
S_{i j}^{c}(\omega)=\sum_{s= \pm} \frac{S_{i j}^{s}}{\omega^{2}+\lambda_{s}^{2}},
$$

where $S_{i j}^{s}$ follows from Eqs. (12) and (15). The total Schottky noise $S_{j}^{\text {Sch }}$ through junction $j$ is a white noise due to the hypothesis of instantaneous tunneling. For a single junction biased by a voltage source, one would get only this term. However, in the spectrum $S_{i j}(\omega)$, interactions do not come into play only through the frequency-dependent term (16). Interactions also modify the values of the terms $\left\langle I_{j \sigma}^{\epsilon}\right\rangle$ determining the Schottky noise. Note that at high frequencies $\omega$ $\gg\left|\lambda_{-}\right|$, we have $S_{i j}(\omega)=\delta_{i j} S_{j}^{\text {Sch }}$. If we furthermore assume $V$ $\gg V_{\max }^{\mathrm{sgn}\left(E_{0}\right)}, S_{j}^{\mathrm{Sch}}=2 e\left|\left\langle I_{j}\right\rangle\right|$ thus $S_{i j}(\omega)$ becomes Poissonian, i.e., $S_{i j}(\omega)=2 e\left|\left\langle I_{i}\right\rangle\right| \delta_{i j}$.

In the three-terminal case studied here, we will be interested in the input Fano factor

$$
F_{2}=\frac{S_{22}(\omega=0)}{2 e\left\langle I_{2}\right\rangle},
$$

and in the output cross-Fano factor

$$
F_{13}=\frac{S_{13}(\omega=0)}{2 e\left\langle I_{2}\right\rangle} .
$$

We also define the resonance voltages

$$
V_{0}=\left|E_{0}\right| \frac{C}{e C_{2}}
$$

and

$$
V_{0}^{+}=\left|E_{0}\right| \frac{C}{e\left(C_{1}+C_{3}\right)} .
$$

Since we consider $V>0$ only, at $B=0$, for $E_{0}$ positive (negative), the dot orbital arrives at resonance with the Fermi level of the input (the outputs) when $V \simeq V_{0}^{+(-)}$. If a magnetic field is applied, each of these voltage resonances is split into two resonances

$$
V_{\uparrow(\downarrow)}=V_{0}+(-) \frac{g \mu_{B} B C}{2 e C_{2}}
$$

and

$$
V_{\uparrow(\downarrow)}^{+}=V_{0}^{+}-(+) \frac{g \mu_{B} B C}{2 e\left(C_{1}+C_{3}\right)},
$$

associated to the $\uparrow(\downarrow)$ levels, respectively, because we consider $B>0$ only. We expect $F_{2}$ and $F_{13}$ to show strong variations for $V \simeq V_{\uparrow(\downarrow)}^{\operatorname{sgn}\left(E_{0}\right)}$.

\section{Time-domain analysis}

The correlation function $\mathcal{C}_{i j}(t)$ can be obtained from the inverse Fourier transform of Eqs. (10), (11), and (16):

$$
\mathcal{C}_{i j}(t)=\delta_{i j} \delta(t) S_{j}^{S c h}+\sum_{s= \pm} \frac{S_{i j}^{S}}{2\left|\lambda_{s}\right|} \exp \left(-|t|\left|\lambda_{s}\right|\right) .
$$

In the sequential tunneling limit, tunneling events occur one by one, thus

$$
\lim _{t \rightarrow 0^{+}} \mathcal{C}_{i j}(t)=-2\left\langle I_{i}\right\rangle\left\langle I_{j}\right\rangle<0 .
$$

Let us first focus on the spin-degenerate case, that is, $\Gamma_{j \uparrow}^{\epsilon}$ $=\Gamma_{j \downarrow}^{\epsilon}$ for $j \in\{1,2,3\}$. In this case, the eigenvectors $v_{+/-}$of $\hat{M}$ correspond to the spin/charge excitations of the system (i.e., $\left.v_{+} \sim[1,-1,0], v_{-} \sim[1,1,-2]\right)$, and $\lambda_{+/-}$to their relaxation rates. This is directly connected to the fact that in the spindegenerate case $S_{i j}^{+}=0$, thus $S_{i j}(\omega)-\delta_{i j} S_{j}^{\text {Sch }}$ is a Lorentzian function and $\mathcal{C}_{i j}(t)-\delta_{i j} \delta(t) S_{j}^{\mathrm{Sch}}=S_{i j}^{-} \exp \left(-|t|\left|\lambda_{-}\right|\right) / 2\left|\lambda_{-}\right|$. This last equation implies that, for any time, $\mathcal{C}_{22}(t)-\delta(t) S_{2}^{\text {Sch }}$ and $\mathcal{C}_{13}(t)$ keep the same sign, which is negative according to Eq. (18). Thus, in the spin-degenerate one-orbital case, $F_{2}$ is always sub-Poissonian and $F_{13}$ always negative. When spin degeneracy is lifted, $v_{+/-}$both become a linear combination of the charge and spin excitations. Thus, having $S_{i j}^{+} \neq 0$ is not forbidden anymore. Equations (17) and (18) altogether with $\left|\lambda_{+}\right|<\left|\lambda_{-}\right|$imply that if $S_{i j}(\omega=0)>\delta_{i j} j_{j}^{\text {Sch }}$, one has $S_{i j}^{-}<0$ and $S_{i j}^{+}>0$. Therefore, in the one-orbital case, a positive sign for $F_{2}-S_{2}^{\text {Sch }} / 2 e\left\langle I_{2}\right\rangle$ and $F_{13}$ can only be due to terms in $\lambda_{+}$.

The results obtained for $\mathcal{C}_{i j}(t)$ can be put in perspective with some fundamental quantities such as the average dwell time $t_{\sigma}$ of spins $\sigma$ on the dot and the average delay $t_{0}$ between the occupancy of the dot by two consecutive electrons. These quantities can be calculated for $\gamma_{\mathrm{sf}}=0$ as

$$
t_{\sigma}=\frac{4 e^{2} \bar{p}_{\sigma}}{\sum_{j} S_{j, \sigma}^{\text {Sch }}}
$$

and

$$
t_{0}=\frac{4 e^{2} \bar{p}_{0}}{\sum_{j} S_{j}^{S c h}} .
$$

The noise reaches its high-voltage limit once $V \gg V_{\max }^{\operatorname{sgn}\left(E_{0}\right)}$ $=\max _{\sigma}\left(V_{\sigma}^{\operatorname{sgn}\left(E_{0}\right)}\right)$ with $V_{\max }^{+}=V_{\downarrow}^{+}$and $V_{\max }^{-}=V_{\uparrow}^{-}$. In this limit, the current transport is unidirectional, i.e., $\left\langle I_{2, \sigma}^{-}\right\rangle=0$ and $\left\langle I_{j, \sigma}^{+}\right\rangle=0$ and for any $j \times \sigma \in\{1,3\} \times\{\uparrow, \downarrow\}$. Thus, Eqs. (19) and (20) lead to $t_{0}=1 / 2 \gamma_{2}$ and $t_{\sigma}=1 /\left(\gamma_{1 \sigma}+\gamma_{3 \sigma}\right)$. The average number 


$$
n_{b}=\frac{S_{2 \uparrow}^{\text {Sch }}}{S_{2 \downarrow}^{\text {Sch }}}
$$

of up spins crossing the input junction between two consecutive down spins for $\gamma_{\mathrm{sf}}=0$, which becomes $n_{b}=I_{2 \uparrow} / I_{2 \downarrow}$ for $V \gg V_{\max }^{\operatorname{sgn}\left(E_{0}\right)}$, is also of importance. It can be used to calculate the average duration

$$
t_{b}=n_{b} t_{\uparrow}+\left(n_{b}+1\right) t_{0}
$$

between the occupation of the dot by two consecutive down spins for $\gamma_{\mathrm{sf}}=0$. In Sec. III C, the analysis of $\mathcal{C}_{i j}(t)$ will be supplemented by simulating numerically the time evolution of the spin $\sigma_{\mathrm{dot}}$ of the dot. As expected, these simulations are in agreement with the results obtained from the master equation approach, but their interest is to allow a visualization of $\sigma_{\mathrm{dot}}(t)$.

\section{Relation between $F_{2}$ and $F_{13}$}

The average input current $\left\langle I_{2}\right\rangle$ and the input Fano factor $F_{2}$ in a three-terminal device correspond to the average current and the Fano factor in a two-terminal device where the output leads 1 and 3 are replaced by an effective output with a net spin-independent tunneling rate $\gamma_{t}=\gamma_{1}+\gamma_{3}$ and with an effective polarization $P_{\text {out }}=\left(\gamma_{1} P_{1}+\gamma_{3} P_{3}\right) / \gamma_{t}$. Then, one fundamental question to answer is whether there is a simple relation between $F_{2}$ and $F_{13}$ in the three-terminal circuit. Charge conservation and the finite dispersion of $\left|\sigma_{\mathrm{dot}}(t)\right|$ lead $\mathrm{to}^{27}$

$$
S_{22}(\omega=0)=S_{11}(\omega=0)+S_{33}(\omega=0)+2 S_{13}(\omega=0) .
$$

At high voltages $V \gg V_{\max }^{\mathrm{sgn}\left(E_{0}\right)}$, the unidirectionality of current transport and the average-currents conservation lead to $S_{2}^{\text {Sch }}$

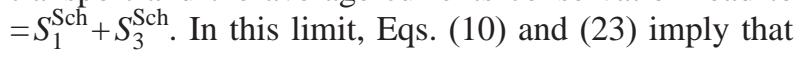

$$
S_{22}^{c}(\omega=0)=S_{11}^{c}(\omega=0)+S_{33}^{c}(\omega=0)+2 S_{13}^{c}(\omega=0) .
$$

Since the voltage bias is the same for leads 1 and 3, we have $\Gamma_{3, \sigma}^{\epsilon} / \Gamma_{1, \sigma}^{\epsilon}=\gamma_{1 \sigma} / \gamma_{3 \sigma}$ for $\epsilon= \pm 1$. Then, in our singly-occupied one-orbital case, Eqs. (12) and (24) lead to

$$
S_{22}^{c}(\omega=0)=\sum_{\sigma, \sigma^{\prime}} \frac{\left(\gamma_{1 \sigma}+\gamma_{3 \sigma}\right)\left(\gamma_{1 \sigma^{\prime}}+\gamma_{3 \sigma^{\prime}}\right)}{\gamma_{1 \sigma} \gamma_{3 \sigma^{\prime}}} S_{1, \sigma, 3, \sigma^{\prime}}^{c}(\omega=0) .
$$

If we furthermore assume $P_{1}=P_{3}$, the ratio $\gamma_{1 \sigma} / \gamma_{3 \sigma}=\gamma_{1} / \gamma_{3}$ is independent of $\sigma$ and

$$
F_{13}=\left(F_{2}-1\right) \frac{\gamma_{1} \gamma_{3}}{\gamma_{t}^{2}}
$$

In summary, for the one-orbital circuit studied here, there exists a simple relation between $F_{2}$ and $F_{13}$ when $P_{1}=P_{3}$ and $V \gg V_{\max }^{\operatorname{sgn}\left(E_{0}\right)}$. Note that the derivation of property (25) requires neither $\gamma_{\mathrm{sf}}=0$, nor $B=0$. On the contrary, the voltage-bias configuration used is crucial. Indeed, if the three leads 1,2 , 3 , were for instance biased at voltages $V, V / 2$ and 0 , respectively, the current transport would not be unidirectional (i.e. right to left in Fig. 1) even in the high $V$ limit. When property (25) is verified, a super-Poissonian (sub-Poissonian) $F_{2}$ is automatically associated with positive (negative) zerofrequency cross correlations. However, Secs. III and IV, which also treat this one-orbital case, illustrate that when $P_{1} \neq P_{3}$ or $V \leqq V_{\max }^{\mathrm{sgn}\left(E_{0}\right)}$, property (25) is not valid anymore, and in particular a super-Poissonian $F_{2}$ can be obtained without a positive $F_{13}$. In Sec. IV B, $F_{2}$ and $F_{13}$ even show variations which are qualitatively different: $F_{13}$ displays a voltage resonance not present in $F_{2}$. Thus, even for the one-orbital quantum dot circuit studied here, the three-terminal problem is in general not trivially connected to the two-terminal problem.

The main ingredients for deriving Eq. (25) are the unidirectionality of current transport and a division of current between the two outputs identical for the two spin directions. One can wonder whether any tunnel-junction circuit with a geometry analogous to that of Fig. 1 satisfies property (25) for $V \gg V_{\max }^{\operatorname{sgn}\left(E_{0}\right)}$ and $P_{1}=P_{3}$. Indeed, it is sometimes the case. For instance, Börlin et al. have studied at $T=0$ a normalmetal island too large to have charging effects, connected, through tunnel junctions, to one superconducting or normal input lead and to two normal output leads with $P_{1}=P_{3}=0$ placed at the same output potential. ${ }^{17}$ For this system, in both the hybrid and the normal cases, a relation analog to Eq. (25) is fulfilled, provided $\gamma_{1} / \gamma_{3}$ is replaced by $g_{1} / g_{3}$, where $g_{1}$ and $g_{3}$ are the conductances of the output junctions. In spite of this, Eq. (25) is not universal even for spin-degenerate tunnel-junction circuits. This can be shown by considering the circuit of Fig. 1, with $B=0, P_{1}=P_{2}=P_{3}=0$ and a twoorbital dot (Sec. V). In this case, the division of currents between the two outputs will generally depend on the orbital considered, because of the different spatial extensions of the orbitals and of the asymmetric positions of the output leads with respect to them. ${ }^{39}$ One has to assume that the division of currents between leads 1 and 3 is independent of the orbital considered in order to recover property (25) at $V \gg V_{\max }^{\mathrm{sgn}\left(E_{0}\right)}$.

\section{E. Influence of screening currents at non-zero frequencies}

The total instantaneous current $I_{j}^{\text {tot }}(t)$ passing through branch $j$ includes the tunneling current $I_{j}(t)$ but also the screening currents needed to guarantee the electrostatic equilibrium of the capacitors after a tunneling event through any junction $i \in\{1,2,3\}$. However, screening currents contribute neither to the average value $\left\langle I_{j}^{\mathrm{tot}}\right\rangle$ of the total current $I_{j}^{\text {tot }}(t)$, i.e., $\left\langle I_{j}^{\text {tot }}\right\rangle=\left\langle I_{j}\right\rangle$, nor to the low frequency part of the total current correlations $S_{i j}^{\text {tot }}(\omega)$, i.e., $S_{i j}^{\text {tot }}(\omega)=S_{i j}(\omega=0)$ for $|\omega|$ $\ll\left|\lambda_{+}\right|$, because, in average, the screening currents due to tunneling through the different junctions compensate each other at zero frequency (see, for instance, Ref. 1). Screening currents contribute to $S_{i j}^{\text {tot }}(\omega)$ only once $S_{i j}(\omega)$ deviates from its zero-frequency limit. In the following, we will assume that the cutoff frequency $\left|\lambda_{+}\right|$is much larger than the inverse of the collective response times associated to the charging of the capacitors. This is equivalent to assuming that, on the time scale on which $\sigma_{\mathrm{dot}}(t)$ varies, any charge variation of the dot triggers instantaneously the screening currents needed for its compensation: 


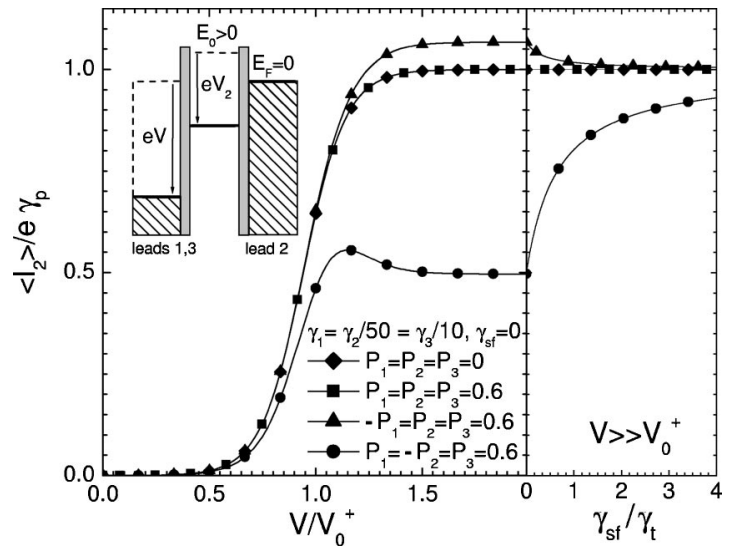

FIG. 2. Left panel: Current-voltage characteristic of the circuit of Fig. 1, for $E_{0}>0, C_{1}=C_{2}=C_{3}, \gamma_{1}=\gamma_{2} / 50=\gamma_{3} / 10, k_{B} T /\left|E_{0}\right|$ $=0.1, B=0$, and different values of lead polarizations. The average current $\left\langle I_{2}\right\rangle$ through lead 2 is plotted in units of its paramagnetic high voltage limit $e \gamma_{p}=2 e \gamma_{2} \gamma_{t} /\left(\gamma_{t}+2 \gamma_{2}\right)$ with $\gamma_{t}=\gamma_{1}+\gamma_{3}$; the voltage in units of the resonance voltage $V_{0}^{+}$(see Sec. II B). For $P_{1}$ $=P_{2}=P_{3}$ (squares), $\left\langle I_{2}\right\rangle$ coincides with the paramagnetic case (diamonds). In the other cases, the high-voltage limit of $\left\langle I_{2}\right\rangle$ can be larger or smaller than the paramagnetic value, depending on the lead polarizations. The inset shows the electrochemical potentials in the circuit. Notation $E_{F}$ refers to the Fermi level in lead 2. (In all the plots, potentials are shown for the case where the dot is empty.) Right panel: Influence of spin-flip scattering in the high-voltage limit $V \gg V_{0}^{+}$. Here, the spin-flip scatterring rate $\gamma_{\mathrm{sf}}$ is expressed in units of $\gamma_{t}$. Spin-flip scattering makes the $\left\langle I_{2}\right\rangle(V)$ curve tend to the paramagnetic one.

$$
I_{j}^{\mathrm{tot}}(t)=I_{j}(t)-\frac{C_{j}}{C} \sum_{i} I_{i}(t)
$$

According to this approximation, the total current correlations $S_{i j}^{\text {tot }}(\omega)$, including screening currents, can be written as

$$
S_{i j}^{\mathrm{tot}}(\omega)=\sum_{n, m}\left(\delta_{i n}-\frac{C_{i}}{C}\right)\left(\delta_{j m}-\frac{C_{j}}{C}\right) S_{n m}(\omega) .
$$

The sign of these total current cross-correlations is not trivial. This problem is addressed in Sec. III E.

\section{ONE-ORBITAL QUANTUM DOT CONNECTED TO FERROMAGNETIC LEADS, IN THE ABSENCE OF A MAGNETIC FIELD}

Here, we focus on the one-orbital case introduced in Sec. II, for $B=0$ and magnetically polarized leads. In the absence of a magnetic field, one single resonance is expected in the voltage characteristics, for $V \simeq V_{0}^{\operatorname{sgn}\left(E_{0}\right)}$. Figures 2-7 show curves for a constant value of the polarization amplitudes $\left|P_{1}\right|=\left|P_{2}\right|=\left|P_{3}\right|=0.6$. This corresponds, for instance, to having the different leads made out of the same ferromagnetic material.

\section{A. Zero-frequency results for $\boldsymbol{E}_{\mathbf{0}}>\mathbf{0}$}

We first consider the case in which the orbital level $E_{0}$ is above the Fermi level of the leads at equilibrium $\left(E_{0}>0\right)$.

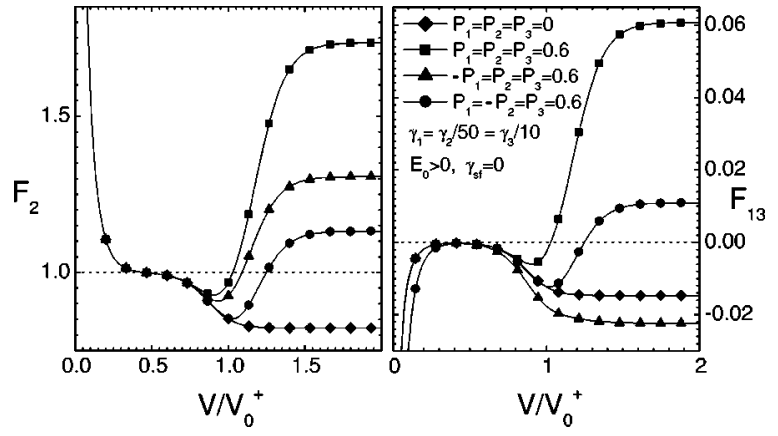

FIG. 3. Input Fano factor $F_{2}=S_{22}(\omega=0) / 2 e I_{2}$ (left panel) and output cross-Fano factor $F_{13}=S_{13}(\omega=0) / 2 e I_{2}$ between leads 1 and 3 (right panel) as a function of the bias voltage $V$ for $\gamma_{\mathrm{sf}}=0$. The curves are shown for the same circuit parameters as in Fig. 2. When $P_{1}=P_{2}=P_{3}$ (squares), $F_{2}$ is different from that of the paramagnetic case (diamonds) in contrast to what happens for $\left\langle I_{2}\right\rangle$. At high enough voltages, the cross correlations are positive in the cases $P_{1}=-P_{2}=P_{3}=0.6$ (circles) and $P_{1}=P_{2}=P_{3}=0.6$. Note that the sign of the cross correlations can be reversed by changing the sign of $P_{1}$. The case $-P_{1}=P_{2}=P_{3}=0.6$ (triangles) illustrates that having a super-Poissonian $F_{2}$ is not sufficient to obtain a positive $F_{13}$.

The typical voltage dependence of the average input current $\left\langle I_{2}\right\rangle$ is shown in the left panel of Fig. 2. This current is exponentially suppressed at low voltages, increases around the voltage $V_{0}^{+}$and saturates at higher voltages. The width on which $\left\langle I_{2}\right\rangle$ varies is of the order of $\Delta V \sim 10 k_{B} T C / e\left(C_{1}\right.$ $\left.+C_{3}\right)$. The high-voltage limit of $\left\langle I_{2}\right\rangle$ depends on the polarizations $P_{i}$ and rates $\gamma_{i}$ but not on the capacitances $C_{i}$ because the tunneling rates saturate at high voltages [see Eq. (2)]. For the paramagnetic case, this limit is

$$
e \gamma_{p}=e \frac{2 \gamma_{2} \gamma_{t}}{\gamma_{t}+2 \gamma_{2}}
$$

In this last expression, $\gamma_{2}$ is weighted by a factor 2 to account for both the populations of up and down spins arriving from the input lead. The rate $\gamma_{t}=\gamma_{1}+\gamma_{3}$ is not weighted by such a factor because there can be only one electron at a time

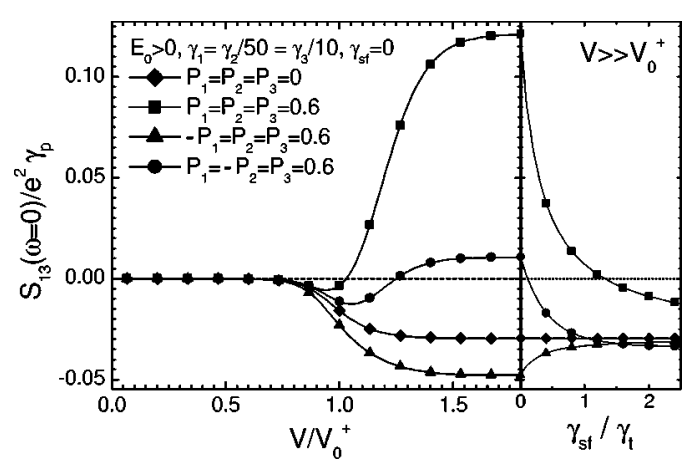

FIG. 4. Zero-frequency cross correlations $S_{13}(\omega=0)$ between leads 1 and 3 as a function of the bias voltage $V$ for $\gamma_{\mathrm{sf}}=0$ (left panel) and as a function of $\gamma_{\mathrm{sf}}$ for $V \gg V_{0}^{+}$(right panel). The curves are shown for the same circuit parameters as in Fig. 2. In the paramagnetic case (diamonds), spin-flip scattering has no effect. In the limit of large $\gamma_{\mathrm{sf}}$, cross correlations tend to the paramagnetic value. 


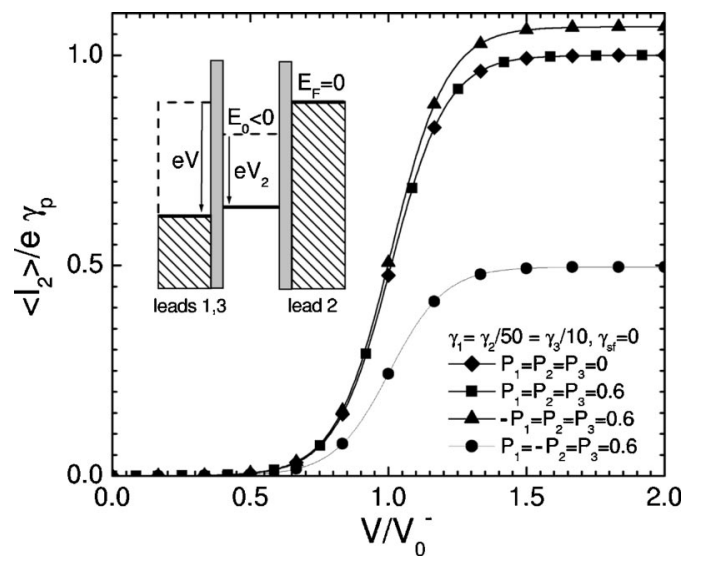

FIG. 5. Current-voltage characteristic of the circuit of Fig. 1 for $E_{0}<0$. The polarizations, tunnel rates, capacitances, and reduced temperature $k_{B} T /\left|E_{0}\right|$ used are the same as in Fig. 2, plotted for $E_{0}>0$. The results differ from the case $E_{0}>0$ only for $V \simeq V_{0}^{-}$.

on the dot, which can tunnel to the output leads with the total rate $\gamma_{t}$. For a sample with magnetic contacts, the highvoltage limit of $\left\langle I_{2}\right\rangle$ can be higher or lower than $e \gamma_{p}$, depending on the parameters considered. Indeed, for $V \gg V_{0}^{+}$, we have $I_{2}\left(P_{1}, P_{2}, P_{3}\right)-I_{2}(0,0,0)=e \gamma_{p} P_{\text {out }}\left\langle\sigma_{\text {dot }}\right\rangle$, where $P_{\text {out }}$ $=\left(P_{1} \gamma_{1}+P_{3} \gamma_{3}\right) / \gamma_{t}$ is the net output lead polarization, and where $\left\langle\sigma_{\text {dot }}\right\rangle=\nu\left(P_{2}-P_{\text {out }}\right)$ is the average spin of the dot. Here, $\nu$ is a positive function of the polarizations, tunneling and scattering rates, which tends to 0 at large $\gamma_{\mathrm{sf}}$. For $P_{1}$ $=P_{2}=P_{3}$, the current is the same as in the paramagnetic case because the populations of spin are matched between the input and the output, thus $\left\langle\sigma_{\mathrm{dot}}\right\rangle=0$. Having a saturation current different from the paramagnetic case requires $P_{\text {out }} \neq 0$ and $\left\langle\sigma_{\text {dot }}\right\rangle \neq 0$. When $\left|P_{\text {out }}\right|>\left|P_{2}\right|$, the high-voltage limit of $\left\langle I_{2}\right\rangle$ is lower than that of the paramagnetic case because the spins in minority at the output block the dot, which leads to a $\left\langle\sigma_{\text {dot }}\right\rangle$ with the same sign as $-P_{\text {out }}$. In this case, $\left\langle I_{2}\right\rangle$ can show negative differential resistance above $V_{0}^{+}$, due to the deblockade of the dot by thermal fluctuations which can send back the blocking spins to electrode 2 for $V \simeq V_{0}^{+}$(Ref. 30) (see the case $P_{1}=-P_{2}=P_{3}=0.6$ in Fig. 2). Spin-flip scattering modifies the $\left\langle I_{2}\right\rangle(V)$ curve once $\gamma_{\mathrm{sf}}$ is of the order of the tunneling rates. It suppresses spin accumulation and makes the $\left\langle I_{2}\right\rangle(V)$ curve tend to the paramagnetic one.

Figure 3 shows $F_{2}$ and $F_{13}$ as a function of $V$ for $\gamma_{\mathrm{sf}}=0$. We also show in Fig. 4 the zero-frequency cross correlations $S_{13}(\omega=0)$ because it is the signal measured in practice. Well below $V_{0}^{+}, S_{13}(\omega=0)$ is exponentially suppressed similar to $\left\langle I_{2}\right\rangle$ because there are very few tunneling events. In this regime, the dot is empty most of the time, and when an electron arrives on the dot, it leaves it with a much higher rate: the electronic transport is limited only by thermally activated tunneling through junction 2. Tunneling events are thus uncorrelated and $F_{2}$ is Poissonian, with a unitary plateau following the thermal divergence $2 k_{B} T / e V$ occurring at $V=0$. For the same reasons, $F_{13}$ displays a zero plateau after a polarization-dependent thermal peak at $V=0$. Around $V$ $\simeq V_{0}^{+}, F_{2}, F_{13}$, and $S_{13}(\omega=0)$ strongly vary. The high-voltage limit depends on tunneling rates and polarizations. In the

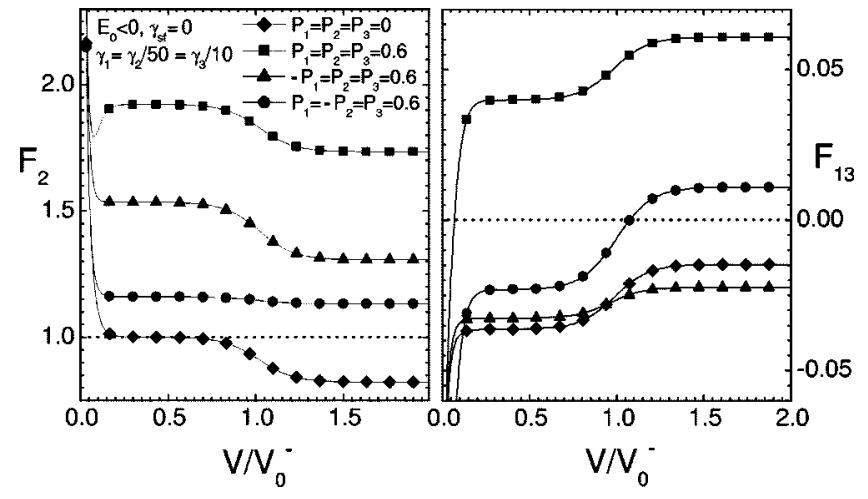

FIG. 6. Input Fano factor $F_{2}$ and cross-Fano factor $F_{13}$ as a function of the bias voltage $V$. The curves are shown for the same circuit parameters as in Fig. 5.

paramagnetic case, the high-voltage limit of $F_{2}$ lies in the interval $[1 / 2,1]$, and that of $F_{13}$ in $[-1 / 8,0]$. In the ferromagnetic case, the high-voltage limit of $F_{2}$ can be either subor super-Poissonian, as already pointed out in the twoterminal case. ${ }^{30}$ Spin accumulation is not a necessary condition for having a super-Poissonian $F_{2}$, as can be seen for $P_{1}=P_{2}=P_{3}$, where $\left\langle\sigma_{\text {dot }}\right\rangle=0$. Negative differential resistance is not necessary either (see case $P_{1}=P_{2}=P_{3}=0.6$ in Figs. 2 and 3). Cross correlations can be either positive or negative depending on the parameters considered, as shown by Figs. 3 and 4. Interestingly, the sign of cross correlations can be switched by reversing the magnetization of one contact. The case $P_{1}=P_{2}=P_{3}=0.6$ of Figs. 3 and 4 corresponds to a superPoissonian $F_{2}$ and a positive $F_{13}$. The case $-P_{1}=P_{2}=P_{3}$ $=0.6$ shows that a super-Poissonian $F_{2}$ is not automatically associated with positive output cross correlations. In this case, the cross correlations are even more negative than in the paramagnetic case. This will be explained physically in Sec. III C.

The effect of spin-flip scattering on $S_{13}(\omega=0)$ is shown in the right panel of Fig. 4. In the paramagnetic case, spin-flip scattering has no effect on $S_{13}(\omega=0)$. In the ferromagnetic case, when $\gamma_{\mathrm{sf}}$ is of the order of the tunneling rates, $S_{13}(\omega$ $=0)$ is modified. In the high- $\gamma_{\mathrm{sf}}$ limit, cross correlations tend

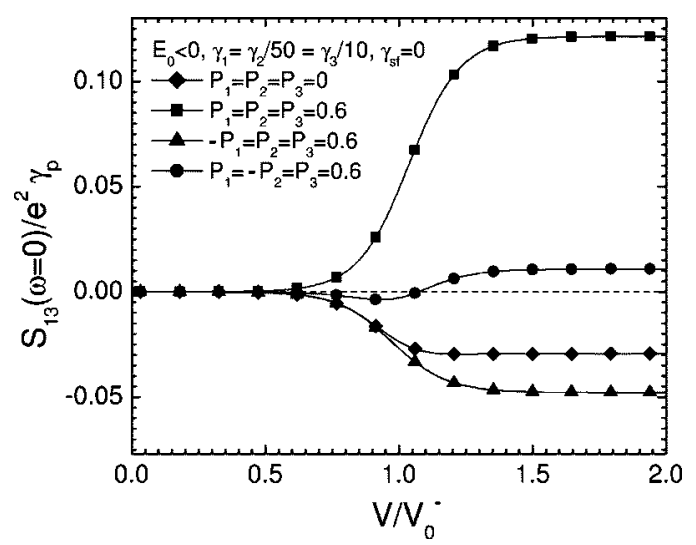

FIG. 7. Zero-frequency current cross correlations $S_{13}(\omega=0)$ between leads 1 and 3 as a function of the bias voltage $V$. The curves are shown for the same circuit parameters as in Fig. 5. 
TABLE II. Top: Zero-frequency output cross correlations $S_{13}(\omega=0)$ and its spin contributions $S_{1 \sigma, 3 \sigma^{\prime}}(\omega$ $=0$ ), division $I_{1 \sigma} / I_{3 \sigma}$ of spin currents between leads 1 and 3 , and average number $n_{b}$ of up spins crossing the input junction between two consecutive down spins, for the high-voltage limit $V \gg V_{0}^{\mathrm{sng}\left(E_{0}\right)}$ of the cases studied in Secs. III A and III B (Figs. 2-7). Bottom: Probabilities $p_{\psi}$ and comparison of the different timescales of the system, for the same parameters. [The summation rules (6) and (10) are not exactly verified by the values given in this table because of the limitation in the number of digits.]

\begin{tabular}{cccccccccc}
\hline \hline case & $S_{13} / e^{2} \gamma_{p}$ & $S_{1 \uparrow, 3 \uparrow} / e^{2} \gamma_{p}$ & $S_{1 \downarrow, 3 \downarrow} / e^{2} \gamma_{p}$ & $S_{1 \uparrow, 3 \downarrow} / e^{2} \gamma_{p}$ & $S_{1 \downarrow, 3 \uparrow} / e^{2} \gamma_{p}$ & $I_{1 \uparrow} / I_{3 \uparrow}$ & $I_{1 \downarrow} / I_{3 \downarrow}$ & $n_{b}$ & \\
\hline$\bullet$ & -0.030 & -0.007 & -0.007 & -0.007 & -0.007 & 0.1 & 0.1 & 1 & \\
$\mathbf{\square}$ & 0.121 & 0.149 & -0.013 & -0.007 & -0.007 & 0.1 & 0.1 & 4 & \\
$\boldsymbol{\Delta}$ & -0.048 & 0.026 & -0.029 & -0.003 & -0.042 & 0.025 & 0.4 & 4 & \\
$\boldsymbol{\bullet}$ & 0.011 & 0.005 & -0.001 & 0.008 & 0.008 & 0.1 & 0.1 & 0.25 & \\
& & & & & & & & & \\
case & $p_{\uparrow}$ & $p_{\downarrow}$ & $p_{0}$ & $\gamma_{p} t_{\uparrow}$ & $\gamma_{p} t_{\downarrow}$ & $\gamma_{p} t_{0}$ & $t_{b} \gamma_{p}$ & $\gamma_{p} /\left|\lambda^{-}\right|$ & $\gamma_{p} /\left|\lambda^{+}\right|$ \\
\hline \multirow{\square}{*}{} & 0.450 & 0.450 & 0.100 & 0.90 & 0.90 & 0.10 & 1.10 & 0.09 & 0.90 \\
$\mathbf{\Delta}$ & 0.450 & 0.450 & 0.099 & 0.56 & 2.25 & 0.10 & 2.75 & 0.09 & 1.46 \\
$\boldsymbol{\bullet}$ & 0.516 & 0.378 & 0.106 & 0.60 & 1.77 & 0.10 & 2.91 & 0.09 & 1.31 \\
\hline \hline
\end{tabular}

to the paramagnetic case for any value of the polarizations. Thus, strong spin-flip scattering suppresses positive cross correlations. However, in practice, it is possible to make quantum dots connected to ferromagnetic leads with spin-flip rates much smaller than the tunneling rates (see for instance Ref. 40). Hence, spin-flip scattering should not be an obstacle for observing positive cross correlations in the quantum-dot circuit studied here.

\section{B. Zero-frequency results for $E_{0}<0$}

We now discuss the case in which the orbital level $E_{0}$ is below the Fermi level of the leads at equilibrium $\left(E_{0}<0\right)$. First, in the low voltage limit in which very few electrons can flow through the device, $\left\langle I_{2}\right\rangle$ and $S_{13}(\omega=0)$ exponentially tend to zero as in the case $E_{0}>0$ (Figs. 5 and 7). However, for $F_{2}$ and $F_{13}$, the results differ (Fig. 6). Above the $2 k_{B} T / e V$ thermal peak, the low voltage plateau of $F_{2}$ is always superPoissonian for $P_{\text {out }} \neq 0$. Above a polarization-dependent thermal peak, $F_{13}$ displays a low voltage plateau which is either negative or positive. This features indicate a correlated transfer of charges in spite of the thermally activated limit. In fact, for $V \ll V_{0}^{-}$, the dot is occupied most of the time and the electronic transport is limited by thermally activated tunneling through the output junctions 1 and 3 . In these conditions, contrarily to what happens for $E_{0}>0$, the polarization of the output leads comes into play even for $V \rightarrow 0$. Indeed, when $P_{\text {out }} \neq 0$, the spins in minority at the output have less chances to leave the dot under the effect of thermal fluctuations. In the intermediate voltage range $V \simeq V_{0}^{-}$, the quantities $\left\langle I_{2}\right\rangle, F_{2}$, $F_{13}$, and $S_{13}(\omega=0)$ differ from the case $E_{0}>0$. However, at $V \gg V_{0}^{-}$, they take the same values as for $E_{0}>0$ and $V \gg V_{0}^{+}$. In this high-voltage limit, the effect of spin flip scattering is identical to that of the case $E_{0}>0$. In particular, the right panels of Figs. 2 and 4 are also valid for $E_{0}<0$.

\section{Interpretation of these zero-frequency results: Dynamical spin blockade}

In this section, we provide a physical explanation for the results of Secs. III A and III B, in the high-voltage limit $V$ $\gg V_{0}^{\operatorname{sgn}\left(E_{0}\right)}$, where the sign of $E_{0}$ does not matter. This analysis relies on the evaluation of quantities defined in Secs. II A and II B (Table II), on numerical simulations of the temporal evolution of the spin $\sigma_{\text {dot }}$ of the dot (Fig. 8) and on plots of

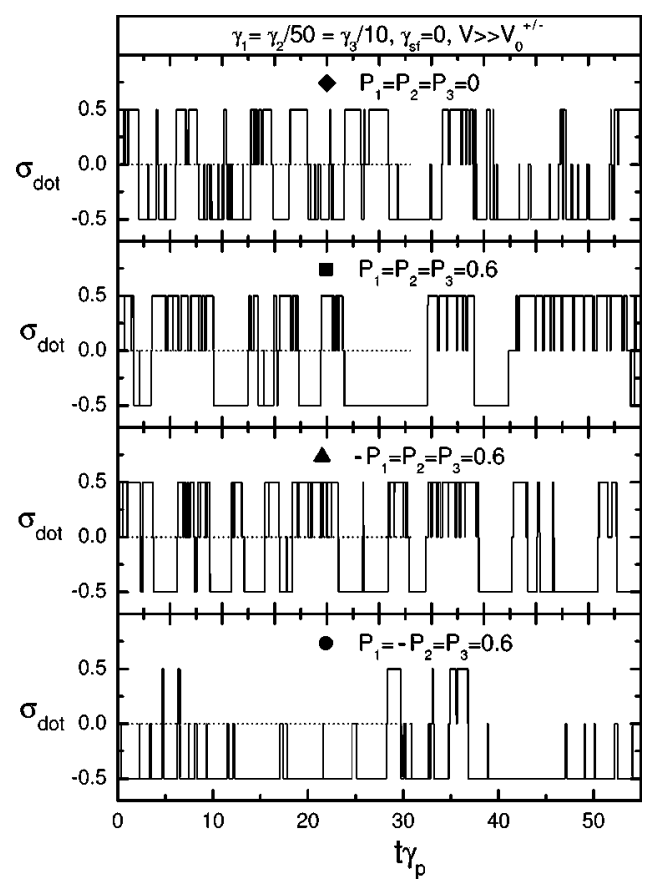

FIG. 8. Numerical simulation of the spin $\sigma_{\text {dot }}$ of the dot as a function of time in the limit $V \gg V_{0}^{\mathrm{sng}\left(E_{0}\right)}$, for the different cases considered in Figs. 2-7. 


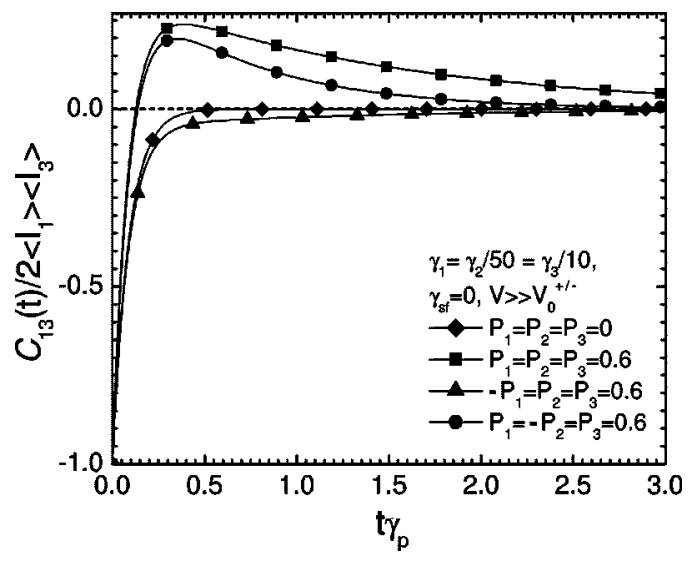

FIG. 9. Time dependence of $C_{13}(t)$ in the limit $V \gg V_{0}^{\mathrm{sng}\left(E_{0}\right)}$, for the different cases considered in Figs. $2-7$. Note that $C_{13}(t)$ is given in units of $-C_{13}(t=0)=2\left\langle I_{1}\right\rangle\left\langle I_{3}\right\rangle$, which depends on the polarization values.

the correlation functions $C_{13}(t)$ (Fig. 9).

Let us first focus on the case $P_{1}=P_{2}=P_{3}=0.6$ (squares in Table II). For these values of lead polarization, up spins are in the majority at the output. Thus, the dwell times of down spins on the dot is longer than that of up spins $\left(t_{\downarrow}>t_{\uparrow}\right.$ in Table II). However, one has $\bar{p}_{\downarrow}=\bar{p}_{\uparrow}$ thus $\left\langle\sigma_{\mathrm{dot}}\right\rangle=0$. This is because $t_{\downarrow}>t_{\uparrow}$ is perfectly compensated by the fact that, due to $P_{2}>0$, up electrons are in the majority in $I_{2}(t)$. Property $t_{\downarrow}>t_{\uparrow}$ suggests that the up spins can flow through the dot only in short time windows where the current transport is not blocked by a down spin. This situation of "dynamical spin blockade" is responsible for a bunching of the tunneling events associated to the up spins, as confirmed by the numerical simulations of $\sigma_{\mathrm{dot}}(t)$ (Fig. 8). The average number of up spins grouped in a "bunch" corresponds to the quantity $n_{b}$ given in Table II (see Ref. 41). On the one hand, the phenomenon of up spins bunching is very strong since, here, $n_{b}=4$. On the other hand, one can see that the positive sign of $S_{13}(\omega=0)$ stems from the up-up correlations [see $S_{1 \uparrow, 3 \uparrow}(\omega=0)>0$ in Table II]. Therefore, one question to answer is whether the positive sign of $S_{13}(\omega=0)$ can be attributed to this bunching of up-spin tunneling events. For that purpose, we have plotted the correlation function $C_{13}(t)$ (Fig. 9) and compared it to the characteristic time scales of the electronic transport. The correlation function $C_{13}(t)$ is negative for times shorter than (approximately) the average delay $t_{0}$ between the occupancy of the dot by two consecutive electrons. Then, $C_{13}(t)$ becomes positive and reaches a maximum at a time comparable to the average delay $t_{0}+t_{\uparrow}$ between the passage of two up spins on the dot. Eventually, $C_{13}(t)$ is strongly decreased at times of the order of the average duration $t_{b}$ of the "bunch" of spins. Hence, the time dependence of $C_{13}(t)$ allows us to attribute the positive value of $S_{13}(\omega$ $=0$ ) to the bunching of tunneling events caused by dynamical spin blockade. The same reasoning can be made to explain the super-Poissonian value of $F_{2}$ (data not shown).

In the case $-P_{1}=P_{2}=P_{3}=0.6$ (triangles in Table II), the temporal evolution of $\sigma_{\mathrm{dot}}$ (see Fig. 8) is qualitatively similar to that of the case $P_{1}=P_{2}=P_{3}=0.6$, thus up-up correlations caused by dynamical spin-blockade lead again to a superPoissonian $F_{2}$. However, less up electrons flow through lead 1 than in the previous case because the polarization $P_{1}$ has been reversed (see $I_{1 \uparrow} / I_{3 \uparrow}$ in Table II). Hence, the positive term $S_{1 \uparrow, 3 \uparrow}(\omega=0)$ is not large enough to lead to a positive $S_{13}(\omega=0)$.

In the case $P_{1}=-P_{2}=P_{3}=0.6$ (circles in Table II), there is still dynamical spin blockade, as shown by $t_{\downarrow}>t_{\uparrow}$ in Table II. This dynamical spin blockade induces again a bunching of the tunneling of up spins [see $S_{1 \uparrow, 3 \uparrow}(\omega=0)>0$ in Table II]. However, the up-up correlations are much weaker than in the $P_{1}=P_{2}=P_{3}=0.6$ case due to the minority of up spins at the input. Another positive contribution to the cross correlations stems from the up-down terms [see $S_{1 \sigma, 3-\sigma}(\omega=0)>0$ in Table II]. In fact, since the average number $n_{b}=0.25$ of up spins passing consecutively through the dot is very low, ${ }^{41}$ we have $t_{b}<t_{\downarrow}$. Then, each up spin is positively correlated to the first down spin preceding him (see Fig. 9). As a result, dynamical spin-blockade now produces a bunching of tunneling events responsible for up-up and up-down correlations. The correlation function $C_{13}(t)$ differs from that of the case $P_{1}=P_{2}=P_{3}=0.6$ in the sense that it decreases more quickly after its maximum, due to the smaller value of $t_{b}$. However, contrarily to the case $P_{1}=P_{2}=P_{3}=0.6$, the decay time of $C_{13}(t)$ is much larger than $t_{b}$, due to large fluctuations in the number of spins per bunch with respect to $n_{b}=0.25$ (Fig. 8). In conclusion, we have seen that in all the cases treated here, the super-Poissonian value of $F_{2}$ and the positive sign of $F_{13}$ can be explained from the dynamical spin-blockade mechanism which induces a bunching of the tunneling events.

\section{Effect of tunneling asymmetry}

We now address the problem of how to choose parameters that favor the observation of positive cross correlations in the ferromagnetic case treated here. First, from Sec. II C, finite lead polarizations are necessary. However, it is possible to get positive cross correlations even if $P_{2}=0$, provided the output of the device is sufficiently polarized. For instance, in the high-voltage limit $V \gg V_{0}^{\mathrm{sgn}\left(E_{0}\right)}$, choosing $P_{1}=P_{3}, P_{2}=0$, and $\gamma_{\mathrm{sf}}=0$ leads to

$$
S_{13}(\omega=0)=\frac{16 e^{2} \gamma_{1} \gamma_{2}^{2} \gamma_{3}\left(1-P_{1}^{2}\right)\left[P_{1}^{2}\left(2 \gamma_{2}+\gamma_{t}\right)-\gamma_{t}\right]}{\gamma_{t}\left[2 \gamma_{2}+\left(1-P_{1}^{2}\right) \gamma_{t}\right]^{3}} \text {. }
$$

In this limit, the current $\left\langle I_{2}\right\rangle$ is not spin polarized, i.e., $\left\langle I_{2, \uparrow}\right\rangle=\left\langle I_{2, \downarrow}\right\rangle$, because up and down spins have the same probability to enter the dot, regardless of what happens at the output. The case where the three electrodes are polarized in the same direction leads to a higher positive $S_{13}(\omega=0)$ because spin accumulation is suppressed $\left(\left\langle\sigma_{\mathrm{dot}}\right\rangle=0\right)$. Indeed, in the high-voltage limit, choosing $P_{1}=P_{2}=P_{3}$ and $\gamma_{\mathrm{sf}}=0$ leads to

$$
S_{13}(\omega=0)=\frac{16 e^{2} \gamma_{1} \gamma_{2}^{2} \gamma_{3}\left[P_{1}^{2}\left(2 \gamma_{2}+\gamma_{t}\right)-\gamma_{t}\right]}{\gamma_{t}\left(2 \gamma_{2}+\gamma_{t}\right)^{3}\left(1-P_{1}^{2}\right)} .
$$

The asymmetry between the tunneling rates $\gamma_{i}$ has a strong influence on the cross correlations. From Eq. (30), the case 


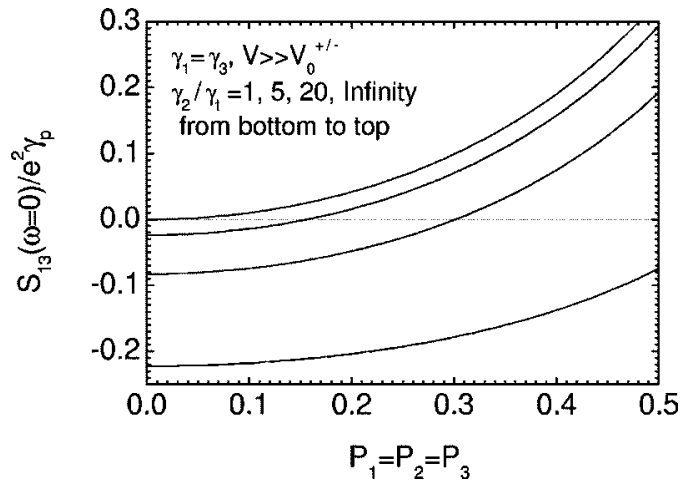

FIG. 10. Influence of the asymmetry between $\gamma_{2}$ and $\gamma_{t}$ on $S_{13}(\omega=0)$, for $V \gg V_{0}^{\operatorname{sgn}\left(E_{0}\right)}, P_{1}=P_{3}=P_{3}, \gamma_{1}=\gamma_{3}$, and $\gamma_{\mathrm{sf}}=0$. According to Eq. (30), for these parameters, $S_{13}(\omega=0)$ is always positive for high enough values of $P_{1}$. Large ratios $\gamma_{2} / \widetilde{\gamma}$ favor positive cross correlations by extending the positivity domain to lower polarization values. In the limit $\gamma_{2} \gg \gamma_{t}$, the curve tends to $S_{13}(\omega=0)$ $=4 \gamma_{1} \gamma_{3} P_{1}^{2} e^{2} / \gamma_{t}\left(1-P_{1}^{2}\right)$.

of symmetric output junctions, i.e., $\gamma_{1}=\gamma_{3}$, is the most favorable configuration for getting a large $S_{13}(\omega=0)>0$ when $P_{1}=P_{2}=P_{3}$. ${ }^{42}$ In addition, choosing large values of $\gamma_{2} / \gamma_{t}$ decreases $\bar{p}_{0}$, which allows to extend the domain of positive cross correlations to smaller values of polarizations (Fig. 10). This is important because ferromagnetic materials are usually not fully polarized (see, for instance, Ref. 43).

\section{E. Finite frequency results}

Equation (16) gives the frequency dependence of $S_{13}(\omega)$. The spectrum $S_{13}(\omega)$ deviates from its zero frequency limit for $\omega \gtrsim\left|\lambda_{+}\right|$. In the case $S_{13}(\omega=0)>0$, properties $\left|\lambda_{+}\right|<\left|\lambda_{-}\right|$, $\mathcal{C}_{13}(t=0)<0, S_{13}^{+}>0$ and $S_{13}^{-}<0$ (see Sec. II C) imply that cross correlations always turn to negative when $\omega$ increases. Then, for frequencies larger than $\left|\lambda_{-}\right|, S_{13}(\omega)$ tends to zero (see Fig. 11).

Equation (27) gives the expression of the total current cross correlations $S_{13}^{\text {tot }}(\omega)$ measured in practice, including the

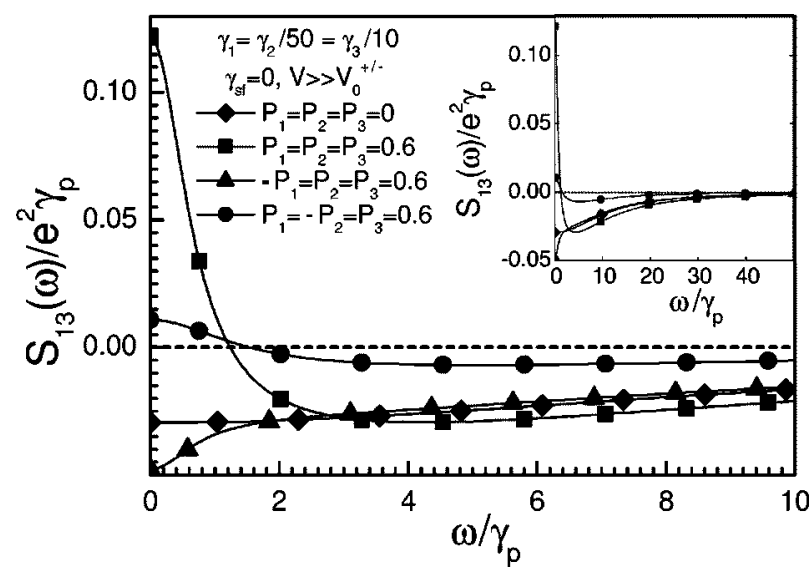

FIG. 11. Frequency dependence of $S_{13}(\omega)$ in the high-voltage limit $V \gg V_{0}^{\operatorname{sgn}\left(E_{0}\right)}$, for the different cases considered in Figs. 2-7. The inset shows the same data for a larger frequency scale. contribution of screening currents. The spectrum $S_{13}^{\text {tot }}(\omega)$ differs from $S_{13}(\omega)$ only for $\omega \gtrsim\left|\lambda_{+}\right|$. At large frequencies $\omega$ $\gg\left|\lambda_{-}\right|, \quad S_{13}^{\text {tot }}(\omega)$ become a linear mixture of the Schottky noises through the three junctions. If we furthermore assume $V \gg V_{0}^{\operatorname{sgn}\left(E_{0}\right)}$, current conservation leads to

$$
\frac{S_{13}^{\mathrm{tot}}(\omega)}{2 e}=\frac{I_{1} C_{3}}{C^{2}}\left(C_{1}-C_{2}-C_{3}\right)+\frac{I_{3} C_{1}}{C^{2}}\left(C_{3}-C_{1}-C_{2}\right) .
$$

This limit depends on the values of $C_{i}$ considered, in contrast to what happens for $S_{i j}(\omega)$. It can be positive as well as negative depending on the values of parameters. For $P_{1}$ $=P_{2}=P_{3}=0.6, \quad \gamma_{1}=\gamma_{2} / 50=\gamma_{3} / 10, \quad C_{1}=C_{2}=C_{3}, \quad$ and $V$ $\gg V_{0}^{\operatorname{sgn}\left(E_{0}\right)}$, one has a crossover from positive to negative cross-correlations as $\omega$ increases $\left[S_{13}^{\text {tot }}(\omega=0) / e^{2} \gamma_{p} \simeq+0.121\right.$ and $\left.S_{13}^{\text {tot }}\left(\omega \gg\left|\lambda_{-}\right|\right) / e^{2} \gamma_{p} \simeq-0.222\right]$. But the opposite situation is also possible. For instance, with $P_{1}=P_{2}=P_{3}=0, \gamma_{1}$ $=\gamma_{2} / 50=\gamma_{3} / 10, C_{1}=C_{2}=C_{3} / 5$, and $V \gg V_{0}^{\operatorname{sgn}\left(E_{0}\right)}$, one has $S_{13}^{\mathrm{tot}}(\omega=0) / e^{2} \gamma_{p} \simeq-0.030<0 \quad$ and $\quad S_{13}^{\mathrm{tot}}\left(\omega \gg\left|\lambda_{-}\right|\right) / e^{2} \gamma_{p}$ $\simeq+0.019>0$. For other positive cross correlations due to screening currents, see Ref. 25 . We recall that the results shown in this section are valid if the Markovian approximation holds, i.e., here $\hbar \omega<\min _{i}\left(\left|E_{0}-e V_{i}\right|\right){ }^{38}$ The results for the correlations of $I_{j}^{\text {tot }}(t)$ are furthermore valid only for $\omega$ larger than the characteristic frequencies associated to the charging of the capacitors (see Sec. II E).

\section{F. Comments}

In spite of the large variety of proposals for getting positive cross correlations in mesoscopic systems, this effect has not been observed experimentally yet. We believe that the mechanism proposed in Sec. III can be implemented with present techniques. For $\gamma_{1}=\gamma_{2} / 10=\gamma_{3}$, the polarizations $P_{1}$ $=P_{2}=P_{3}=0.4$ typical for Co (Ref. 43) lead to positive cross correlations of the order of $S_{13} / e^{2} \gamma_{\text {tot }} \simeq 0.08$. With $\gamma_{p}$ $\simeq 5 \mathrm{GHz}$, this corresponds to a current noise level of $10^{-29} \mathrm{~A}^{2} \mathrm{~s}$. The maximum differential conductance of the sample depends on temperature: $\quad d\left\langle I_{2}\right\rangle / d V \sim e^{2} \gamma_{p}\left(C_{1}\right.$ $\left.+C_{3}\right) / 5 k_{B} T C$. Assuming that $T=20 \mathrm{mK}$ and $C_{1}=C_{2}=C_{3}$, one obtains $\left(d\left\langle I_{2}\right\rangle / d V\right)^{-1} \sim h / e^{2}$. This leads to a voltage noise level measurable with existing voltage noise-amplification techniques. ${ }^{29,44}$

One difficulty of this experiment is connecting three leads to a very small structure. We believe that a multiwall carbon nanotube (MWNT) contacted by ferromagnetic leads could be an interesting candidate for implementing a three-terminal device. The question of whether a MWNT splits into two quantum dots when three contacts are evaporated on top it is still open. However, given that the intrinsic level spacing of a MWNT connected to two leads seems to be determined by its total length rather than by the separation between the leads, ${ }^{45}$ a three-terminal quantum dot structure seems feasible. In addition, it has been demonstrated experimentally that contacting ferromagnetic leads to a MWNT is possible. ${ }^{46}$

Interestingly, a different mechanism, proposed by Sauret and Feinberg, can also lead to positive current cross correlations in a quantum-dot circuit connected to ferromagnetic leads. ${ }^{47}$ This work also considers current transport through 
one single orbital of the dot. For certain bias voltages large enough to allow a double occupation of this orbital, the Pauli principle induces positive correlations between up and down spins. This so-called mechanism of "opposite-spin bunching" is antagonist to our mechanism of dynamical spin blockade which requires that the orbital can be only singly occupied. However, with both mechanisms, positive cross correlations can be obtained only when the two spin channels do not transport current independently, i.e., when charging effects are relevant. ${ }^{48}$ We point out that in the three-terminal geometry of Fig. 1, the opposite-spin bunching proposed by Sauret et al. allows us to get positive output cross correlations in spite of a sub-Poissonian input Fano factor. This feature, added to our findings, shows that positive output cross correlations and a super-Poissonian input Fano factor can be obtained separately for a quantum dot connected to ferromagnetic leads. Nevertheless, the opposite-spin bunching proposed by Sauret et al. can lead to positive cross correlations between the total currents through leads 1 and 3 only when the output leads are strongly polarized in opposite directions, in order to filter the weak up-down positive cross correlations induced by this effect. In practice, this is very difficult to achieve with usual ferromagnetic materials. ${ }^{43}$

Note that the dynamical spin blockade studied in this article is unrelated with another mechanism called "spin blockade," observed in many semiconductor quantum dots experiments (see Ref. 49, and references therein). This other spinblockade refers to the suppression of peaks expected in the $I-V$ characteristics of a quantum dot for independent single electron states, but not observed due to quantum mechanical spin selection rules.

\section{ONE-ORBITAL QUANTUM DOT IN A MAGNETIC FIELD, CONNECTED TO THREE PARAMAGNETIC LEADS}

In view of the experimental difficulties for connecting ferromagnetic leads to semiconductor quantum dots, ${ }^{50}$ the ques-

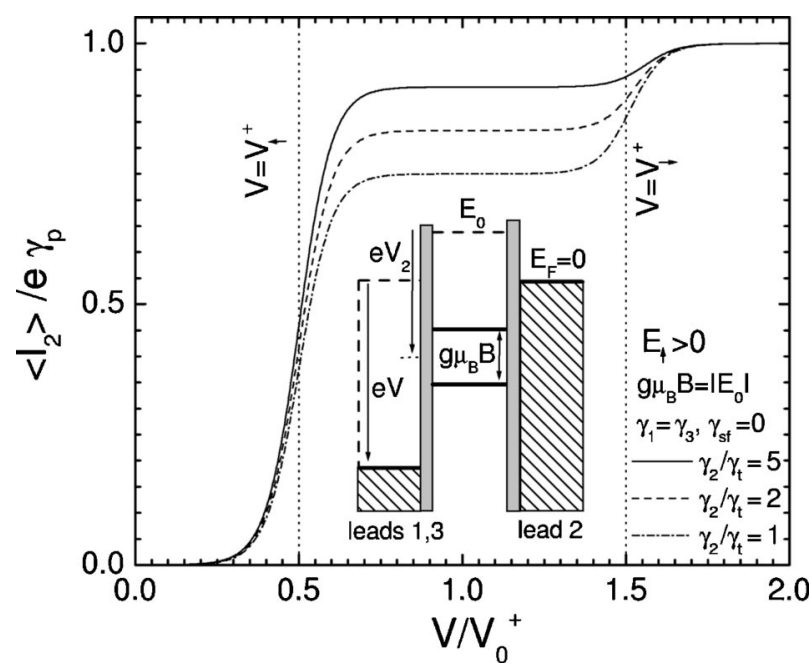

FIG. 12. Average current $\left\langle I_{2}\right\rangle$ as a function of the bias voltage $V$ for $E_{0}>0, P_{1}=P_{2}=P_{3}=0, C_{1}=C_{2}=C_{3}, \quad \gamma_{1}=\gamma_{3}, k_{B} T /\left|E_{0}\right|=0.05$, $g \mu_{B} B /\left|E_{0}\right|=1$, and different values of $\gamma_{2} / \gamma_{t}$. These curves display two steps, for $V \simeq V_{\uparrow}^{+}$and $V \simeq V_{\downarrow}^{+}$. The inset shows electrochemical potentials in the circuit. tion of whether it is possible to obtain positive cross correlations without using ferromagnetic leads is of great interest. We thus consider in Secs. IV A and IV B the one-orbital case introduced in Sec. II, with $P_{1}=P_{2}=P_{3}=0$ and $B \neq 0$.

At $B \neq 0$, two resonances are expected $a$ priori in the voltage characteristics, for $V \simeq V_{\uparrow}^{\operatorname{sgn}\left(E_{0}\right)}$ and $V \simeq V_{\downarrow}^{\operatorname{sgn}\left(E_{0}\right)}$. The limit $V \gg V_{\max }^{\mathrm{sgn}\left(E_{0}\right)}$ and $\gamma_{\mathrm{sf}}=0$ is the same as in the $B=0$ case because the tunneling rates saturate at high voltages. In particular, from Eqs. (25), (28), and (30), we have in this limit

$$
\begin{gathered}
F_{2}=\frac{4 \gamma_{2}^{2}+\gamma_{t}^{2}}{\left(\gamma_{t}+2 \gamma_{2}\right)^{2}}, \\
F_{13}=-\frac{4 \gamma_{1} \gamma_{2} \gamma_{3}}{\gamma_{t}\left(\gamma_{t}+2 \gamma_{2}\right)^{2}} .
\end{gathered}
$$

This means that here, a super-Poissonian $F_{2}$ and positive cross correlations can appear only at lower voltages, for which the cases $E_{0}>0$ and $E_{0}<0$ differ significantly. Note that due to $P_{1}=P_{2}=P_{3}=0$, one obtains from Eqs. (4) and (12):

$$
S_{13}=\frac{\gamma_{1} \gamma_{3}}{\gamma_{t}} \mathcal{F}\left(\frac{\gamma_{2}}{\gamma_{t}}, \frac{\gamma_{\uparrow \downarrow}}{\gamma_{t}}, \frac{\gamma_{\downarrow \uparrow}}{\gamma_{t}}, \frac{E_{0}}{T}, \frac{V}{T}, \frac{B}{T}\right) .
$$

According to Eq. (32), for a constant value of $\gamma_{t}, \gamma_{1}=\gamma_{3}$ allows to maximize $\left|S_{13}\right|$. Therefore, in this section, we will plot curves for $\gamma_{1}=\gamma_{3}$.

\section{A. Zero-frequency results for $E_{0}>0$}

We first briefly comment the case in which the two Zeeman sublevels are above the Fermi energy at equilibrium (i.e., $E_{\uparrow(\downarrow)}>0$ ). The current and noise voltage characteristics obtained in this situation were already discussed in Ref. 51 for the two-terminal case. As in Sec. III A, for $V<V_{\uparrow}^{+},\left\langle I_{2}\right\rangle$

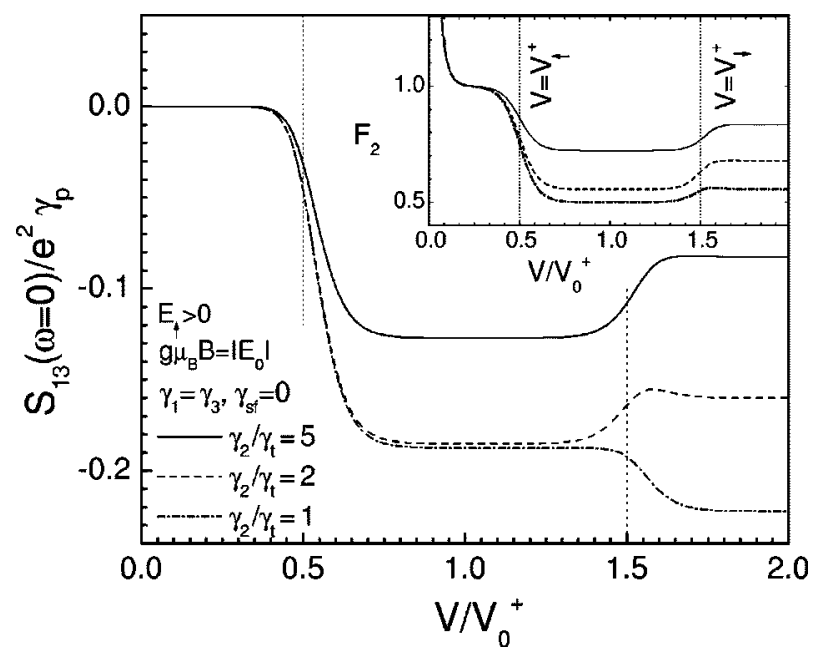

FIG. 13. Zero-frequency current cross correlations $S_{13}(\omega=0)$ between leads 1 and 3 as a function of the bias voltage $V$ for the same circuit parameters as in Fig. 12. Inset: Fano factor $F_{2}$. These curves display two steps, for $V \simeq V_{\uparrow}^{+}$and $V \simeq V_{\downarrow}^{+}$. Above the thermal peak, for $\gamma_{\mathrm{sf}}=0$, one has $F_{2} \leqslant 1$ and $S_{13}(\omega=0) \leqslant 0$ for any values of the parameters. 


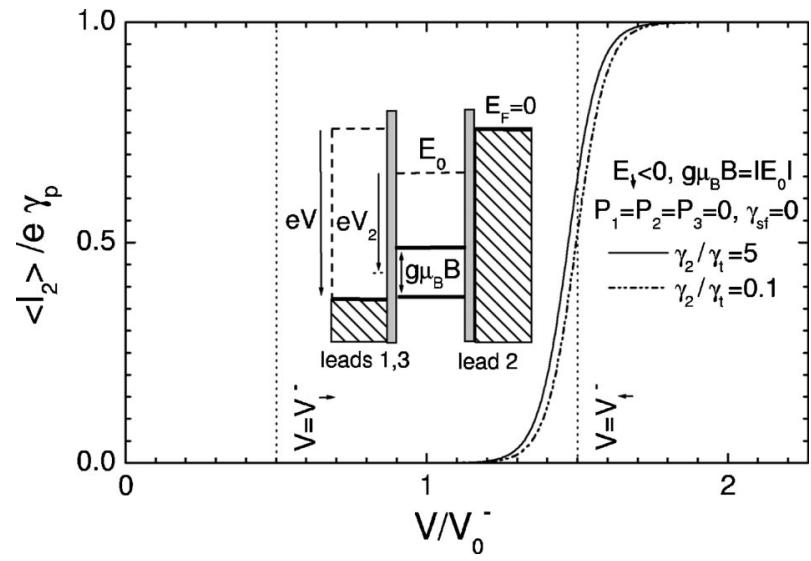

FIG. 14. Current-voltage characteristic of the circuit of Fig. 1 for $E_{0}<0, P_{1}=P_{2}=P_{3}=0, C_{1}=C_{2}=C_{3}, \gamma_{1}=\gamma_{3}, k_{B} T /\left|E_{0}\right|=0.05$, $g \mu_{B} B /\left|E_{0}\right|=1$, and different values of the asymmetry $\gamma_{2} / \gamma_{t}$ between the input and the output. These curves display only one step, for $V \simeq V_{\uparrow}^{-}$. The inset shows the electrochemical potentials in the circuit.

and $S_{13}(\omega=0)$ are exponentially small and $F_{2}$ is Poissonian with a thermal peak at $V \rightarrow 0$, followed by a unitary plateau (Figs. 12 and 13). Then, the curves $\left\langle I_{2}\right\rangle, F_{2}$ and $S_{13}(\omega=0)$ show two steps corresponding to $V \simeq V_{\uparrow}^{+}$and then $V \simeq V^{+}$. We have verified analytically that, above the thermal peak, for $\gamma_{\mathrm{sf}}=0$, one has $F_{2} \leqslant 1$ and $F_{13} \leqslant 0$ for any values of the parameters. For $V<V_{\downarrow}^{+}$, the current $\left\langle I_{2}\right\rangle$ is spin polarized, an effect which allows to do spin filtering with a nearly $100 \%$ efficiency. ${ }^{52,53}$

\section{B. Zero-frequency results for $E_{0}<0$}

Below, we focus on the case in which the two Zeeman sublevels are below the Fermi energy at equilibrium (i.e., $\left.E_{\uparrow(\downarrow)}<0\right)$. To our knowledge, the current noise in this configuration has never been studied before, even for a twoterminal device. We will first study analytically what happens above the thermal peak, i.e., $e V \gg k_{B} T$. In this limit, one can write the tunneling rates as $\Gamma_{2 \sigma}^{+}=\gamma_{2}, \Gamma_{2 \sigma}^{-}=0, \Gamma_{1(3) \uparrow}^{-}$ $=x \gamma_{1(3)}, \quad \Gamma_{1(3) \uparrow}^{+}=(1-x) \gamma_{1(3)}, \quad \Gamma_{1(3) \downarrow}^{-}=y \gamma_{1(3)}, \quad$ and $\quad \Gamma_{1(3) \downarrow}^{+}$ $=(1-y) \gamma_{1(3)}$, where $x=1 /\left\{1+\exp \left[-\left(E_{\uparrow}-e V_{1}\right) / k_{B} T\right]\right\}$ and $y$ $=1 /\left\{1+\exp \left[-\left(E_{\downarrow}-e V_{1}\right) / k_{B} T\right]\right\}$. The hypothesis $B>0$ implies that $x<y$. First, for $V<V_{\downarrow}$, we have $x \rightarrow 0$ and $y \rightarrow 0$. Then, the parameters $x$ and $y$ go from 0 to 1 while the voltage increases. For $V=V_{\downarrow}$ i.e., $y=1 / 2$, the upper Zeeman sublevel is at resonance with the Fermi level of the output leads 1 and 3. Then, for $V=V_{\uparrow}$ i.e. $x=1 / 2$, the lower Zeeman sublevel is at resonance with the outputs, as represented by the level diagram in Fig. 14.

The assumptions made on the rates lead to

$$
F_{13}=\frac{\gamma_{1} \gamma_{3}}{\gamma_{2} \gamma_{t}^{2}}\left[\gamma_{2}\left(F_{2}-1\right)+\gamma_{t}(x+y-2)\right] \text {. }
$$

In Sec. II, we have shown that relation (25) between $F_{2}$ and $F_{13}$ is always valid at high voltages (i.e, $x, y \sim 1$ here) for the single-orbital problem with $P_{1}=P_{2}=P_{3}$. But this demonstra- tion does not take into account the symmetries that the problem takes for certain particular cases. Here, from Eq. (33), $P_{1}=P_{2}=P_{3}=0$ implies that property (25) is also valid at any $V$ above the thermal peak when $\gamma_{2} \gg \gamma_{t}$.

The inequality $t_{\downarrow}=1 / y \gamma_{t} \neq t_{\uparrow}=1 / x \gamma_{t}$ for $x \neq y$ suggests the possibility of obtaining again dynamical spin blockade. To study the situation accurately, we will consider the simple limit $k_{B} T \ll g \mu_{B} B$, i.e., the up-spins channel starts to conduct for voltages such that down spin can flow only from the right to the left. This means that for the first voltage transition $V$ $\simeq V_{\downarrow}$ (i.e., $y \simeq 1 / 2$ ), we have $x \ll 1$ and it is enough to consider low order developments of $\left\langle I_{2}\right\rangle, F_{2}$, and $F_{13}$ with respect to $x$ :

$$
\begin{gathered}
\left\langle I_{2}\right\rangle=\frac{2 e \gamma_{2} \gamma_{t} x}{\tilde{\gamma}+\gamma_{2}}+o(x)^{2}, \\
F_{2}=\frac{\gamma_{t}+3 \gamma_{2}}{\gamma_{t}+\gamma_{2}}+o(x),
\end{gathered}
$$

and

$$
F_{13}=\frac{\gamma_{1} \gamma_{3}\left[\left(2 \gamma_{2}^{2}-\gamma_{t}\left(\gamma_{t}+\gamma_{2}\right)(2-y)\right]\right.}{\left(\gamma_{t}+\gamma_{2}\right) \gamma_{2} \gamma_{t}^{2}}+o(x)
$$

for $\gamma_{\mathrm{sf}}=0$. Transport through the upper level is energetically allowed for $y>1 / 2$. However, since we have assumed $x$ $\ll 1$, from Eq. (34), $\left\langle I_{2}\right\rangle$ remains very small throughout the $V \simeq V_{\downarrow}$ transition: the dot is blocked by up spins, thus down spins cannot cross the dot. Even if the current is very low, this leads to dynamical spin blockade and thus to a superPoissonian $F_{2}$, except in the limit $\gamma_{t} \gg \gamma_{2}$ [see Eq. (35)]. Accordingly, $F_{13}$ can be positive for certain tunneling rate asymmetries [Eq. (36)]. The factor $F_{13}$ shows a step around $V \simeq V_{\downarrow}^{-}$, due to the $y$ dependence in Eq. (36), whereas $F_{2}$ is constant throughout the $V \simeq V_{\downarrow}$ transition. This implies a redistribution of the zero-frequency noise between $S_{11}(\omega=0)$, $S_{33}(\omega=0)$, and $S_{13}(\omega=0)$ when the threshold $V=V_{\downarrow}$ is crossed [see Eq. (23)]. The absence of step for $F_{2}$ can be attributed to the unidirectionality of tunneling through junction 2. Indeed, $x \rightarrow 0$ means that $F_{2}$ depends only on $p_{0}$ and $G_{0, \uparrow(\downarrow)}$ [see Eqs. (7) and (12)]. Now, for $V \simeq V_{\downarrow}$, the contribution of these terms is independent of $y$ (and thus on $V$ ) at first order in $x$, because $\bar{p}_{0}$ and $G_{0, \uparrow(\downarrow)}$ are already forced to very low values due to the $x \rightarrow 0$ hypothesis. On the contrary, $F_{13}$ also depends on $\bar{p}_{\uparrow, \downarrow}$ and $G_{\sigma, 0}$ with $\sigma \in\{\uparrow, \downarrow, 0\}$. For $x$ $\rightarrow 0$, these last terms depend strongly on $y$.

For $k_{B} T \ll g \mu_{B} B$, the second possible voltage transition $V \simeq V_{\uparrow}$ (i.e., $x \simeq 1 / 2$ ) can be described by taking the limit $y$ $=1$, where

$$
\begin{gathered}
\left\langle I_{2}\right\rangle=\frac{2 e x \gamma_{2} \gamma_{t}}{\gamma_{t}+\gamma_{2}(1+x)}, \\
F_{2}=1+\frac{2 \gamma_{2}\left[\gamma_{t}(1-3 x)+(1-x)^{2} \gamma_{2}\right]}{\left[\gamma_{t}+\gamma_{2}(1+x)\right]^{2}}
\end{gathered}
$$

and 


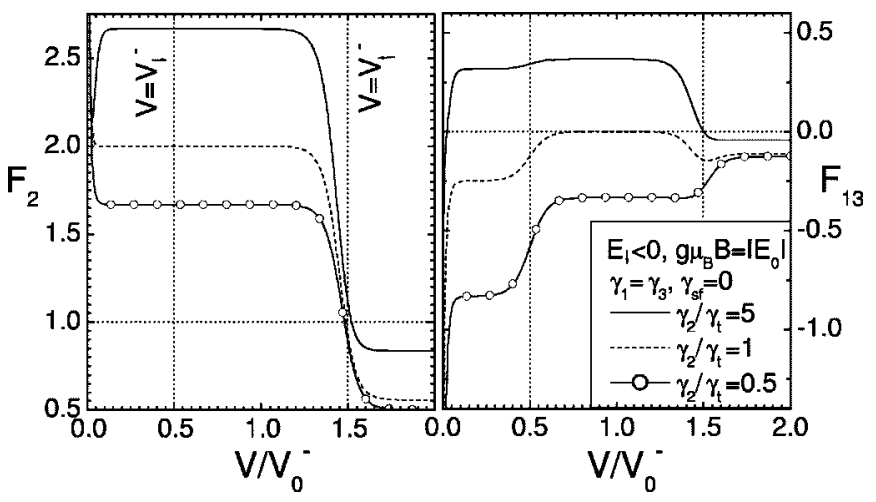

FIG. 15. Input Fano factor $F_{2}$ (left panel) and output cross-Fano factor $F_{13}$ (right panel) as a function of the bias voltage $V$, for the same circuit parameters as in Fig. 14. In all curves $\gamma_{\mathrm{sf}}=0$. The Fano factor $F_{2}$ shows only one step for $V \simeq V_{\uparrow}^{-}$whereas $F_{13}$ shows two steps, for $V \simeq V_{\downarrow}$ and $V \simeq V_{\uparrow} . F_{2}$ can be super-Poissonian and $F_{13}$ positive for certain values of $\gamma_{2} / \gamma_{t}$ and $V$ (see text).

$$
\begin{aligned}
F_{13}= & \frac{\gamma_{1} \gamma_{3}}{\gamma_{t}^{2}} \frac{1}{\gamma_{2}\left[\gamma_{t}+(1+x) \gamma_{2}\right]^{2}}\left[2(1-x)^{2} \gamma_{2}^{3}\right. \\
& \left.+\left(1-7 x+x^{2}+x^{3}\right) \gamma_{2}^{2} \gamma_{t}-2\left(1-x^{2}\right) \gamma_{2} \gamma_{t}^{2}-(1-x) \gamma_{t}^{3}\right]
\end{aligned}
$$

for $\gamma_{\mathrm{sf}}=0$. Around $V \simeq V_{\uparrow}$, the blockade of the dot by up spins is partially lifted and transport through both levels is allowed. The average input current $\left\langle I_{2}\right\rangle$ thus increases with voltage (i.e., with $x$ ) [see Eq. (37)]. On the opposite of what happens in Sec. IV A, the average current $\left\langle I_{2}\right\rangle$ is not spin polarized because up and down spin have the same probability to enter the dot. The factors $F_{2}$ and $F_{13}$ both show a step through the $V \simeq V_{\uparrow}$ transition (as indicated by their $x$ dependency) and tend at high voltages to the usual sub-Poissonian values.

We now turn to the discussion of the general results displayed in Figs. 14-17, obtained from an exact treatment of the full Master equation. Figure 14 shows the full voltage dependence of $\left\langle I_{2}\right\rangle$. As expected from Eqs. (34) and (37), this current shows a single step at $V \simeq V_{\uparrow}$, an effect observed experimentally. ${ }^{54-56}$ The width on which $\left\langle I_{2}\right\rangle$ varies is of the order of $\Delta V \sim 10 k_{B} T C / e C_{2}$, whereas the position of the step varies only slightly with the asymmetry of the junctions (the maximal variation is about $\Delta V^{\prime} \sim 0.7 k_{B} T C / e C_{2}$ ).

The left panel of Fig. 15 shows the voltage dependence of $F_{2}$. The low-voltage divergence of $F_{2}$ is again a result of the dominating thermal noise in the limit $k_{B} T \gtrsim e V$. As expected from Eqs. (35) and (38), $F_{2}$ shows one single step at $V$ $\simeq V_{\uparrow}$, surrounded by two plateaus, and the low voltage plateau of $F_{2}$ is super-Poissonian because $t_{\downarrow}>t_{\uparrow}$ induces dynamical spin blockade (except in the limit $\gamma_{t} \gg \gamma_{2}$ ). The right panel of Fig. 15 shows the voltage dependence of $F_{13}$. As expected from Eqs. (36) and (39), $F_{13}$ shows two steps at $V \simeq V_{\downarrow}$ and then $V \simeq V_{\uparrow}^{-}$, and $F_{13}$ can be positive at low voltages, due again to $t_{\downarrow}>t_{\uparrow}$. The first plateau displayed by $F_{13}$ is positive for $\gamma_{2}>\gamma_{t}(1+\sqrt{5}) / 2$ and the second for $\gamma_{2}>\gamma_{t}$, as can be seen from Eq. (36). The high-voltage plateau of $F_{13}$ is

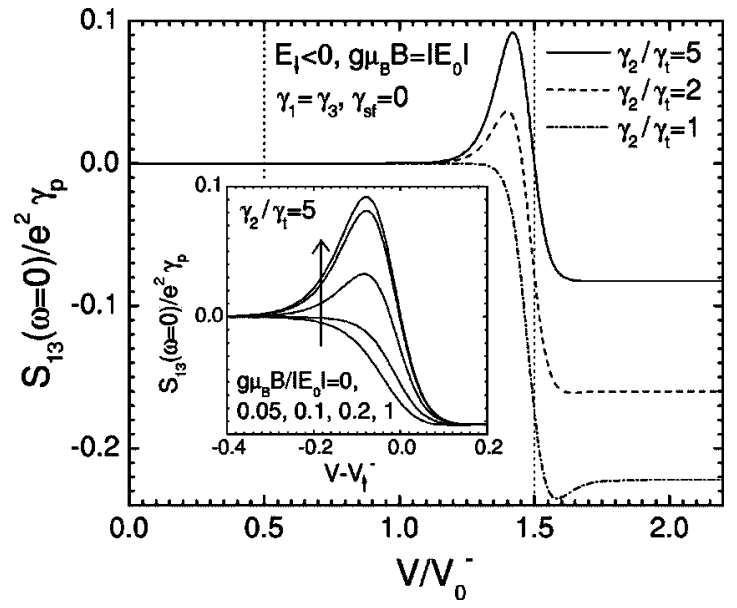

FIG. 16. Zero-frequency current cross-correlations $S_{13}(\omega=0)$ between leads 1 and 3 as a function of the bias voltage $V$, for the same circuit parameters as in Fig. 14 and different values of junction asymmetry. The inset shows the effect of a magnetic field $B$ for $\gamma_{2} / \gamma_{t}=5$ and $\gamma_{\mathrm{sf}}=0$.

negative because $t_{\uparrow}$ and $t_{\downarrow}$ tend to the same value. Note that the case $\gamma_{2} / \gamma_{t}=1 / 2$ and $V_{\downarrow}<V<V_{\uparrow}$ is one more illustration that it is possible to have $F_{2}$ super-Poissonian and $F_{13}<0$.

It is also interesting to look at $S_{13}(\omega=0)$ which is the signal measured in practice (Fig. 16). Similar to $\left\langle I_{2}\right\rangle$, the cross correlations $S_{13}(\omega=0)$ are exponentially small for $V$ $\simeq V_{\downarrow}^{-}$, thus the first voltage step of $F_{13}$ is not visible on the scale of Fig. 16. Cross correlations have a significant variation around the voltage $V \simeq V_{\uparrow}^{-}$, for which the blockade of the dot by up spins is partially lifted. When $\gamma_{2}>\gamma_{t}$, this variation consists of a positive peak consistent with the positive plateau found for $F_{13}$. The maximum positive $S_{13}(\omega=0)$ obtained at this peak is of the same order as the maximum $S_{13}(\omega=0)$ predicted in the ferromagnetic case for comparable junction asymmetries (see Sec. III F). Note that the height of the peak is independent of temperature as long as Eq. (1) is fulfilled, whereas its width, which is approximately $\Delta V$, depends on temperature. At high voltages $S_{13}(\omega=0)$ is always

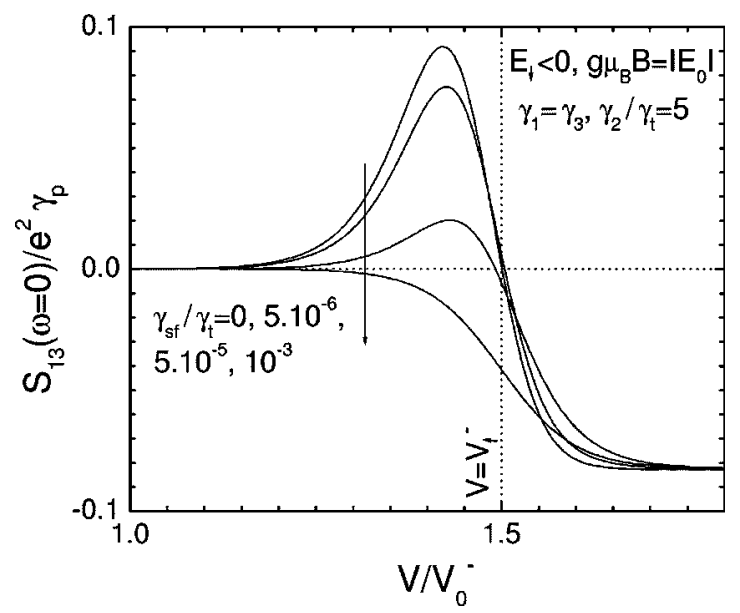

FIG. 17. Effect of spin flip scattering on the current cross correlations between leads 1 and 3 for the same circuit parameters as in Fig. 16 and $\gamma_{2} / \gamma_{t}=5$. 
negative, in agreement with the behavior of $F_{13}$.

Since the positive cross correlations found in this work are due to dynamical spin blockade, we expect a strong dependence on the magnetic field. The inset of Fig. 16 shows the voltage dependence of $S_{13}(\omega=0)$ around the step $V_{\uparrow}^{-}$, for a fixed temperature, a tunneling asymmetry $\gamma_{2} / \gamma_{t}=5$, and various magnetic fields. The amplitude of the positive peak first increases with $B$ and then saturates once the Zeeman splitting of the levels is much larger than the thermal smearing of the resonances (i.e., $g \mu_{B} B \geqslant 8 k_{B} T$ ). The peak then simply shifts to larger bias voltages $V$ while $B$ increases. Figure 17 shows the effect of spin-flip scattering on the cross correlations. Spin flips modify the positive peak of $S_{13}(\omega=0)$ when $\Gamma_{\uparrow \downarrow}=\gamma_{\text {sf }} \exp \left(g \mu_{B} B / 2 k_{B} T\right) \sim \gamma_{i}$, see Eq. (4). As expected, a strong spin-flip scattering suppresses all spin effects and turns the positive cros correlations to negative. It is thus preferable to use a $B$ not larger than $8 k_{B} T$ when spin flip scattering is critical.

\section{Comments}

There is a strong qualitative difference between the ferromagnetic case of Sec. III and the $B \neq 0$ case of Sec. IV: in Sec. IV we have obtained positive cross correlations in the form of a peak around a resonance voltage whereas in Sec. III, positive cross correlations reach their maximum above the resonance voltage.

In practice, we can imagine to tune the bias voltage $V$ such that different orbital levels will transport current successively while the gate voltage of the dot is swept, leading to an effective $E_{0}$ oscillating between positive and negative values. In this situation, the results of Secs. IV A and V B indicate the possibility of having the sign of $S_{13}(\omega=0)$ which oscillates with the gate voltage.

MWNT's could be possible candidates for observing this effect. However, lateral semiconductor quantum dots seem even more attractive. The fabrication technology of lateral semiconductor quantum dots allows to engineer more than two leads just by adjusting a lithography pattern (see, for instance, Ref. 39). Another advantage of these structures is that the asymmetry of the tunnel junctions, which is very critical for getting dynamical spin blockade, can be controlled just by changing the voltage of the gates delimiting them. In addition, it has been shown that the spin-flip rate can be very low in semiconductor quantum dots. ${ }^{56,57}$ However, implementing the model of Sec. IV requires that the leads can be considered as unpolarized, which is not obvious in these systems if the magnetic field is not applied locally to the dot but to the whole circuit. In certain cases, the magnetic field can induce a significant spin polarization at the edges of the two-dimensional electron gas, leading to different net tunneling rates $\gamma_{j, \uparrow}$ and $\gamma_{j, \downarrow}$ for up and down spins. ${ }^{53,58,59}$ In an extremely simplified approach, we have taken this effect into account with finite polarizations $P_{1}=P_{2}=P_{3}$ with the same sign as $B$ (see Fig. 18). The positive peak of $S_{13}(\omega$ $=0)$ is suppressed while $P_{1}$ increases because the tunneling rates of spins which blocked the dot for $P_{1}=P_{2}=P_{3}=0$ increase. However, this positive peak is replaced by a highvoltage positive limit simply identical to that of Sec. III for

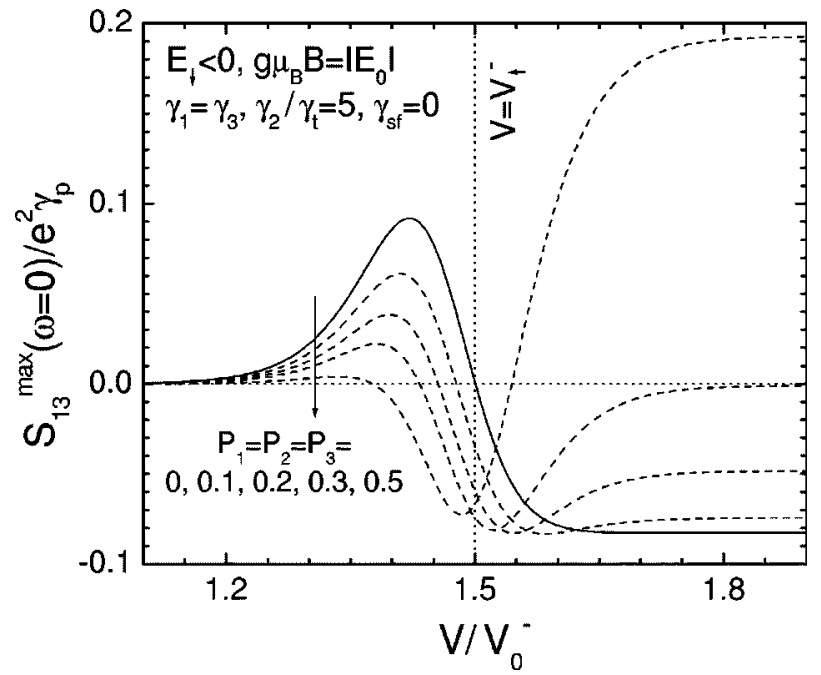

FIG. 18. Zero-frequency current cross correlations between leads 1 and 3 as a function of the bias voltage for the same circuit parameters as in Fig. 16, $\gamma_{2} / \gamma_{t}=5$ and $\gamma_{\mathrm{sf}}=0$. The full line corresponds to the case $P_{1}=P_{2}=P_{3}=0$ shown in Fig. 16 and the dashed lines to finite values of $P_{1}=P_{2}=P_{3}$.

the corresponding polarizations. Note that for semiconductor quantum dots in the few electron regime, the time evolution of $\left|\sigma_{\text {dot }}\right|$ can be measured by coupling the dot to a single electron transistor or a quantum point contact. ${ }^{60-63}$ In the high-voltage limit where current transport is unidirectional, studying the statistics of $\left|\sigma_{\mathrm{dot}}(t)\right|$ would give a direct access to $S_{22}(\omega)$ for currents too low to be measured with standard techniques.

\section{TWO-ORBITAL SPIN-DEGENERATE QUANTUM DOT CIRCUIT}

\section{A. Mapping onto the one-orbital non-spin-degenerate case}

We now consider the quantum dot circuit of Fig. 1 with $V>0$, connected to paramagnetic leads $\left(P_{1}=P_{2}=P_{3}=0\right)$ and with no magnetic field $(B=0)$. We assume that two different orbitals levels $a$ and $b$ of the dot are accessible for current transport (see Fig. 19), but we still consider that the dot cannot be doubly occupied. We define $\gamma_{j, \text { orb }}$ as the net tunneling rate between lead $j$ and the orbital orb $\in\{a, b\}$. This

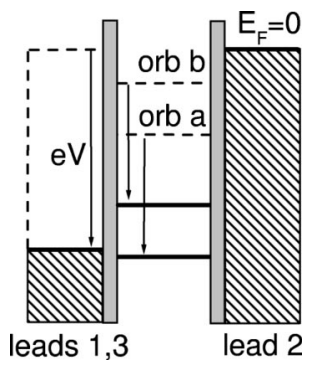

FIG. 19. Electrochemical potentials for a quantum dot connected to three paramagnetic leads and subject to no magnetic field, with two different orbitals levels $a$ and $b$ accessible for current transport. 
problem is spin degenerate and can thus be treated without the spin degree of freedom, which is replaced by the orbital degree of freedom. The rate for an electron to tunnel between lead $j$ and the orbital level "orb" in direction $\epsilon$ is $\Gamma_{j \text {,orb }}^{\epsilon}$ $=\gamma_{j, \text { orb }} /\left(1+\exp \left[\epsilon\left(E_{\text {orb }}-e V_{j}\right) / k_{B} T\right]\right)$, where $E_{\text {orb }}$ is the intrinsic energy of the orbital level "orb." This problem can be treated in the sequential tunneling limit with a Master equation analog to Eq. (3). There is in fact a direct mapping between this problem and that described in Sec. II. We will assume that $E_{a}<E_{b}$, so that the orbitals $a$ and $b$ will play the roles of the Zeeman sublevels $\uparrow$ and $\downarrow$ of Sec. II, where $B>0$. One has to replace the parameters of the previous problem by

$$
\begin{gathered}
E_{\uparrow(\downarrow)} \rightarrow E_{a(b)}, \\
\gamma_{j} \rightarrow \frac{\gamma_{j, a}+\gamma_{j, b}}{2}, \\
P_{j} \rightarrow \widetilde{P}_{j}=\frac{\gamma_{j, a}-\gamma_{j, b}}{\gamma_{j, a}+\gamma_{j, b}}, \\
\gamma_{\uparrow \downarrow(\downarrow \uparrow)} \rightarrow \gamma_{a b(b a)} .
\end{gathered}
$$

This mapping shows that one can obtain a superPoissonian $F_{2}$ or a positive $F_{13}$ in this two-orbital system. This result demonstrates that interactions can lead to zerofrequency positive cross correlations in a normal quantum dot circuit even without lifting spin degeneracy. Note that in practice, $\gamma_{j, a}=\gamma_{j, b}$ is not obvious because of the different spatial extensions of the orbitals (see, for instance, Refs. 39 and 53). This problem is thus equivalent to a one-orbital problem with $B \neq 0$ and with finite effective polarizations $\widetilde{P_{1}}, \widetilde{P_{2}}, \widetilde{P_{3}}$ which can be close to \pm 1 . Positive cross correlations can be expected either at the resonance associated to level $b$ (for $E_{b}<0$ ) or in the plateau following this resonance, depending on the parameters (see, for instance, Figs. 18).

\section{B. Comments}

In the one-orbital ferromagnetic case, we have shown that the simple relation (25) between $F_{2}$ and $F_{13}$ is valid in the high-voltage limit only when $P_{1}=P_{3}$. Therefore, according to the mapping indicated in Sec. V A, in the two-orbital case, relation (25) is valid in the high-voltage limit only if $\widetilde{P_{1}}$ $=\widetilde{P_{3}}$, i.e., $\gamma_{1, a} / \gamma_{3, a}=\gamma_{1, b} / \gamma_{3, b}$. Hence, the conditions of validity of property (25) found in Sec. II D for the one-orbital system (i.e., same polarization for the two output leads and high-voltage limit) cannot be generalized to the two-orbital case.

In the spin-degenerate case treated here, positive cross correlations stem from the partial blockade of an electronic channel by another one, thus we suggest to call this effect dynamical channel blockade. This effect should be observable in semiconductor quantum dots. The advantage of taking $B=0$ is that the problem of spurious lead polarization evoked in Sec. IV is suppressed. When $\mathrm{eV} \gg\left|E_{b}+E_{C}\right|$ and $\gamma_{\mathrm{sf}}=0$, the two channels conduct current independently, thus dynamical channel blockade is suppressed and the positive cross correlations disappear [see, Eq. (40) and Ref. 48]. When $\Delta E \ll k_{B} T$, cross correlations are always negative in a spin-degenerate three-terminal quantum dot placed in the sequential tunneling limit. ${ }^{28}$ Therefore, our hypothesis $\Delta E$ $\gg k_{B} T$ is also necessary to obtain positive cross correlations in this device. In fact, when $\Delta E \ll k_{B} T$, the electron leaving the dot at a given time is not necessarily the one which entered the dot just before, in spite of $\mathrm{eV} \ll E_{C}$ : channel effects are suppressed.

Note that a super-Poissonian Fano factor can also be obtained in a spin-degenerate circuit based on two biterminal quantum dots (or localized impurity states) placed in parallel and coupled electrostatically to each other. ${ }^{64-66}$ If one of the dots is charged, the other cannot transport current because of the Coulomb repulsion. The dot which changes its occupancy with a lower rate modulates the current through the other one, which leads to a dynamical channel-blockade analogous to what we found. The possibility to get positive cross correlations in these systems was not investigated, but Sec. V of the present article suggests it.

\section{CONCLUSION}

We have considered noise in a three-terminal quantum dot operated as a beam splitter. In this system, a superPoissonian input Fano factor is not equivalent to zerofrequency positive output cross correlations. We have studied three different ways to get these two effects, due to the mechanism of dynamical channel blockade. The first two strategies consist in involving only one orbital of the dot in the electronic transport and lifting spin degeneracy, either by using ferromagnetic leads or by applying a magnetic field to the dot. We have furthermore shown that lifting spin degeneracy is not necessary anymore when two orbitals of the dot are involved in the current transport. These results show that one can get zero-frequency positive cross correlations due to interactions inside a beam splitter circuit even if this is a spin-degenerate normal fermionic circuit with a perfect voltage bias.

\section{ACKNOWLEDGMENTS}

We thank H. A. Engel, K. Ensslin, M. Governale, H. Grabert, R. Hanson, T. Kontos, R. Leturcq, B. Reulet, I. Safi, P. Samuelsson, and B. Trauzettel for interesting discussions. We are particularly indebted to $\mathrm{M}$. Büttiker for raising the question which led us to consider the case treated in Sec. V. This work was financially supported by the RTN Spintronics, by the Swiss NSF and the NCCR Nanoscience. 
${ }^{1}$ Ya. M. Blanter and M. Büttiker, Phys. Rep. 336, 1 (2000).

${ }^{2}$ Quantum Noise in Mesoscopic Physics, edited by Yu. V. Nazarov (Kluwer, Dordrecht, 2003).

${ }^{3}$ V. A. Khlus, Sov. Phys. JETP 66, 1243 (1987).

${ }^{4}$ G. B. Lesovik, JETP Lett. 49, 592 (1989).

${ }^{5}$ M. Büttiker, Phys. Rev. Lett. 65, 2901 (1990).

${ }^{6}$ M. Büttiker, Phys. Rev. B 46, 12485 (1992).

${ }^{7}$ M. Henny, S. Oberholzer, C. Strunk, T. Heinzel, K. Ensslin, M. Holland, and C. Schönenberger, Science 284, 296 (1999).

${ }^{8}$ W. D. Oliver, J. Kim, R. C. Liu, and Y. Yamamoto, Science 284, 299 (1999)

${ }^{9}$ S. Oberholzer, M. Henny, C. Strunk, C. Schönenberger, T. Heinzel, K. Ensslin, and M. Holland, Physica E (Amsterdam) 6, 314 (2000).

${ }^{10}$ See the article of M. Büttiker, in Ref. 2.

${ }^{11}$ T. Martin, Phys. Lett. A 220, 137 (1996).

${ }^{12}$ M. P. Anantram and S. Datta, Phys. Rev. B 53, 16390 (1996).

${ }^{13}$ J. Torres and T. Martin, Eur. Phys. J. B 12, 319 (1999).

${ }^{14}$ T. Gramespacher and M. Büttiker, Phys. Rev. B 61, 8125 (2000).

${ }^{15}$ J. Torres, T. Martin, and G. B. Lesovik, Phys. Rev. B 63, 134517 (2001).

${ }^{16}$ M. Shechter, Y. Imry, and Y. Levinson, Phys. Rev. B 64, 224513 (2001).

${ }^{17}$ J. Börlin, W. Belzig, and C. Bruder, Phys. Rev. Lett. 88, 197001 (2002).

${ }^{18}$ P. Samuelsson and M. Büttiker, Phys. Rev. Lett. 89, 046601 (2002).

${ }^{19}$ P. Samuelsson and M. Büttiker, Phys. Rev. B 66, 201306 (2002).

${ }^{20}$ F. Taddei and R. Fazio, Phys. Rev. B 65, 134522 (2002).

${ }^{21}$ D. Sánchez, R. López, P. Samuelsson, and M. Büttiker, Phys. Rev. B 68, 214501 (2003).

${ }^{22}$ G. Bignon, M. Houzet, F. Pistolesi, and F. W. J. Hekking, condmat/0310349 (unpublished).

${ }^{23}$ C. Texier and M. Büttiker, Phys. Rev. B 62, 7454 (2000).

${ }^{24}$ M. Gattobigio, G. Iannaccone, and M. Macucci, Phys. Rev. B 65, 115337 (2002).

${ }^{25}$ A. M. Martin and M. Büttiker, Phys. Rev. Lett. 84, 3386 (2000).

${ }^{26}$ I. Safi, P. Devillard, and T. Martin, Phys. Rev. Lett. 86, 4628 (2001).

${ }^{27}$ A. N. Korotkov, Phys. Rev. B 49, 10381 (1994); S. Hershfield, J. H. Davies, P. Hyldgaard, C. J. Stanton, and J. W. Wilkins, ibid. 47, 1967 (1993); U. Hanke, Yu., M. Galperin, K. A. Chao, and N. Zou, ibid. 48, 17209 (1993).

${ }^{28}$ D. A. Bagrets and Yu. V. Nazarov, Phys. Rev. B 67, 085316 (2003).

${ }^{29}$ H. Birk, M. J. M. de Jong, and C. Schönenberger, Phys. Rev. Lett. 75, 1610 (1995); H. Birk, K. Oostveen, and C. Schönenberger, Rev. Sci. Instrum. 67, 2977 (1996).

${ }^{30}$ B. R. Bulka, J. Martinek, G. Michalek, and J. Barnas, Phys. Rev. B 60, 12246 (1999); B. R. Bulka, ibid. 62, 1186 (2000).

${ }^{31}$ E. V. Sukhorukov, G. Burkard, and D. Loss, Phys. Rev. B 63, 125315 (2001).

${ }^{32}$ D. V. Averin, in Macroscopic Quantum Coherence and Quantum Computing, edited by D. V. Averin, B. Ruggiero, and P. Silvestrini (Kluwer, Dordrecht, 2001); cond-mat/0010052.

${ }^{33}$ F. Yamaguchi and K. Kawamura, Physica B 227, 116 (1996); G.-H. Ding and T.-K. Ng, Phys. Rev. B 56, R15 521 (1997); Y. Meir and A. Golub, Phys. Rev. Lett. 88, 116802 (2002).

${ }^{34}$ A. Cottet, W. Belzig, and C. Bruder, Phys. Rev. Lett. 92, 206801 (2004).
${ }^{35}$ A. Cottet and W. Belzig, Europhys. Lett. 66, 405 (2004).

${ }^{36}$ W. Belzig and M. Zareyan Phys. Rev. B 69, 140407(R) (2004).

${ }^{37}$ M. Julliere, Phys. Lett. 54A, 225 (1975).

${ }^{38}$ H. A. Engel and D. Loss cond-mat/0312107 (unpublished).

${ }^{39}$ R. Leturcq, D. Graf, T. Ihn, K. Ensslin, D. Driscoll, and A. C. Gossard, Europhys. Lett. 67, 439 (2004).

${ }^{40}$ M. M. Deshmukh and D. C. Ralph, Phys. Rev. Lett. 89, 266803 (2002); M. M. Deshmukh, E. Bonet, A. N. Pasupathy, and D. C. Ralph, Phys. Rev. B 65, 073301 (2002).

${ }^{41}$ Note that $n_{b}=I_{2 \uparrow} / I_{2 \downarrow}$ includes bunches with no up spins, i.e. two down spins passing consecutively. Thus $n_{b}$ can be smaller than 1 .

${ }^{42}$ Note that despite this, we have chosen to show in Secs. II A and II B curves for the case $\gamma_{3} / \gamma_{1}=10$ because it allows to get, just by reversing the polarization of one output lead, both the cases where $S_{13}(\omega=0)$ is positive or negative while $F_{2}$ is superPoissonian.

${ }^{43}$ R. J. Soulen Jr., J. M. Byers, M. S. Osofsky, B. Nadgorny, T. Ambrose, S. F. Cheng, P. R. Broussard, C. T. Tanaka, J. Nowak, J. S. Moodera, A. Barry, and J. M. D. Coey, Science 282, 85 (1998).

${ }^{44}$ D. C. Glattli, P. Jacques, A. Kumar, P. Pari, and L. Saminadayar, J. Appl. Phys. 81, 7350 (1997).

${ }^{45}$ M. R. Buitelaar, A. Bachtold, T. Nussbaumer, M. Iqbal, and C. Schönenberger, Phys. Rev. Lett. 88, 156801 (2002).

${ }^{46}$ K. Tsukagoshi, B. W. Alphenaar, and H. Ago, Nature (London) 401, 572 (1999).

${ }^{47}$ O. Sauret and D. Feinberg, Phys. Rev. Lett. 92, 106601 (2004).

${ }^{48}$ In the one-orbital case, positive cross-correlations can be obtained only if the tunneling rates for one spin direction depend on the occupation of the other spin level. When charging effects are not relevant, i.e., $V \gg\left|E_{\uparrow}+E_{C}\right| C /\left(C_{1}+C_{3}\right)$ for $E_{0}>0$ or $V \gg \mid E_{\downarrow}$ $+E_{C} \mid C / C_{2}$ for $E_{0}<0$, the tunneling rates become independent of the occupation of the dot and one can check that for $\gamma_{\mathrm{sf}}=0$, $S_{13}(\omega=0)$ is always negative for any polarization of the leads 1 , $2,3$.

${ }^{49}$ A. K. Hüttel, H. Qin, A. W. Holleitner, R. H. Blick, K. Neumaier, D. Weinmann, K. Eberl, and J. P. Kotthaus, Europhys. Lett. 62, 712 (2003).

${ }^{50}$ G. Schmidt, D. Ferrand, L. W. Molenkamp, A. T. Filip, and B. J. van Wees, Phys. Rev. B 62, R4790 (2000); A. Khaetskii, J. C. Egues, D. Loss, C. Gould, G. Schmidt, and L. W. Molenkamp, cond-mat/0312705 (unpublished).

${ }^{51}$ A. Thielmann, M. H. Hettler, J. König, and G. Schön, Phys. Rev. B 68, 115105 (2003).

${ }^{52}$ P. Recher, E. V. Sukhorukov, and D. Loss, Phys. Rev. Lett. 85, 1962 (2000).

${ }^{53}$ R. Hanson, L. M. K. Vandersypen, L. H. Willems van Beveren, J. M. Elzerman, I. T. Vink, and L. P. Kouwenhoven, cond-mat/ 0311414 (unpublished).

${ }^{54}$ D. C. Ralph, C. T. Black, and M. Tinkham, Phys. Rev. Lett. 74, 3241 (1995); ibid. 78, 4087 (1997).

${ }^{55}$ D. H. Cobden, M. Bockrath, P. L. McEuen, A. G. Rinzler, and R. E. Smalley, Phys. Rev. Lett. 81, 681 (1998); D. H. Cobden and J. Nygard, ibid. 89, 046803 (2002).

${ }^{56}$ R. Hanson, B. Witkamp, L. M. K. Vandersypen, L. H. Willems van Beveren, J. M. Elzerman, and L. P. Kouwenhoven, Phys. Rev. Lett. 91, 196802 (2003).

${ }^{57}$ A. V. Khaetskii and Yu. V. Nazarov, Phys. Rev. B 61, 12639 (2000); 64, 125316 (2001); S. I. Erlingsson and Yu. V. Nazarov, 
ibid. 66, 155327 (2002).

${ }^{58}$ M. Ciorga, M. Pioro-Ladriere, P. Zawadski, P. Hawrylak, and A. S. Sachrajda, Appl. Phys. Lett. 80, 2177 (2002).

${ }^{59}$ M. C. Rogge, C. Fuhner, U. F. Keyser, and R. J. Haug, Appl. Phys. Lett. 85, 606 (2004).

${ }^{60}$ W. Lu, Z. Ji, L. Pfeiffer, K. W. West, and A. J. Rimberg, Nature (London) 423, 422 (2003).

${ }^{61}$ M. Field, C. G. Smith, M. Pepper, D. A. Ritchie, J. E. F. Frost, G. A. C. Jones, and D. G. Hasko, Phys. Rev. Lett. 70, 1311 (1993).

${ }^{62}$ L. M. K. Vandersypen, J. M. Elzerman, R. N. Schouten, L. H. Willems van Beveren, R. Hanson, and L. P. Kouwenhoven, cond-mat/0407121 (unpublished).

${ }^{63}$ R. Schleser, E. Ruh, T. Ihn, K. Ensslin, D. C. Driscoll, and A. C. Gossard, cond-mat/0406568 (unpublished).

${ }^{64}$ S. S. Safonov, A. K. Savchenko, D. A. Bagrets, O. N. Jouravlev, Y. V. Nazarov, E. H. Linfield, and D. A. Ritchie, Phys. Rev. Lett. 91, 136801 (2003).

${ }^{65}$ G. Kießlich, A. Wacker, and E. Schöll, Phys. Rev. B 68, 125320 (2003).

${ }^{66}$ A. Nauen, F. Hohls, J. Könemann, and R. J. Haug, Phys. Rev. B 69, 113316 (2004). 